\title{
On the impact of swirl on the growth of coherent structures
}

\author{
K. Oberleithner ${ }^{1} \dagger$, C. O. Paschereit ${ }^{2}$ and I. Wygnanski ${ }^{3}$ \\ ${ }^{1}$ Laboratory for Turbulence Research in Aerospace and Combustion, Monash University, Clayton, \\ VIC 3800, Australia \\ ${ }^{2}$ Institut für Strömungsmechanik und Technische Akustik, HFI, Müller-Breslau Straße 8, D-10623 Berlin, \\ Germany \\ ${ }^{3}$ Department of Aerospace and Mechanical Engineering, PO Box 210119, Tucson, AZ 85721, USA
}

(Received 2 May 2013; revised 29 September 2013; accepted 14 December 2013; first published online 7 February 2014)

Spatial linear stability analysis is applied to the mean flow of a turbulent swirling jet at swirl intensities below the onset of vortex breakdown. The aim of this work is to predict the dominant coherent flow structure, their driving instabilities and how they are affected by swirl. At the nozzle exit, the swirling jet promotes shear instabilities and, less unstable, centrifugal instabilities. The latter stabilize shortly downstream of the nozzle, contributing very little to the formation of coherent structures. The shear mode remains unstable throughout generating coherent structures that scale with the axial shear-layer thickness. The most amplified mode in the nearfield is a co-winding double-helical mode rotating slowly in counter-direction to the swirl. This gives rise to the formation of slowly rotating and stationary large-scale coherent structures, which explains the asymmetries in the mean flows often encountered in swirling jet experiments. The co-winding single-helical mode at high rotation rate dominates the farfield of the swirling jet in replacement of the co- and counter-winding bending modes dominating the non-swirling jet. Moreover, swirl is found to significantly affect the streamwise phase velocity of the helical modes rendering this flow as highly dispersive and insensitive to intermodal interactions, which explains the absence of vortex pairing observed in previous investigations. The stability analysis is validated through hot-wire measurements of the flow excited at a single helical mode and of the flow perturbed by a time- and space-discrete pulse. The experimental results confirm the predicted mode selection and corresponding streamwise growth rates and phase velocities.

Key words: absolute/convective instability, turbulent flows, wakes/jets

\section{Introduction}

Turbulent swirling jets represent a flow configuration that is both easy to generate in an experiment and astonishingly rich in physical problems. The complexity of this flow configuration poses a great challenge to fundamental research and often serves as a benchmark for recent theoretical concepts. Moreover, swirling flows are commonly

†Email address for correspondence: kilian.oberleithner@pi.tu-berlin.de 
used in industrial applications where efficient turbulent mixing is required, due to the ability of swirl to enhance turbulent production and shear-layer spreading. The great importance of swirling flows for the combustion industry is reflected by the large number of related publications (see the review articles of McManus, Poinsot \& Candel 1993; Gutmark, Schadow \& Yu 1995; Knowles \& Saddington 2006; Huang \& Yang 2009, and references therein). Its relevance and frequent application demands a profound understanding of its governing flow physics.

During the last two decades, various ideas have arisen to explain the enhanced turbulent mixing in swirling flows. There is a common consensus that the vortical structures in the swirled shear layers, which differ from those found in non-swirling jets, must be the driving force for the enhanced turbulent production and entrainment rate. However, the characteristics and the sources of the flow structures that reside in swirled shear layers are still unclear. It is known that with the addition of swirl, the flow promotes shear instabilities and centrifugal instabilities, but their ability to enhance jet spreading is still a controversial issue. Linear stability analysis based on swirling jet models reveal that centrifugal instabilities become successively destabilized with increasing swirl (Martin \& Meiburg 1996, 1998; Lu \& Lele 1999; Loiseleux, Delbende \& Huerre 2000; Gallaire \& Chomaz 2003b; Müller \& Kleiser 2008). Several investigators suggest these instabilities, which promote flow structures at smaller scales than shear instabilities, to be the cause for the enhanced jet spreading (Mehta, Wood \& Clausen 1991; Panda \& McLaughlin 1994; Cutler, Kraus \& Levey 1995; Wu, Ma \& Zhou 2006). This stands in contrast to the experimental observation of Naughton, Cattafesta \& Settles (1997), who found swirl-enhanced mixing for a centrifugally stable profile, which is confirmed by the numerical studies of $\mathrm{Hu}$, Sun \& Yin (2001a), Hu et al. (2001b). These authors concluded that centrifugal instability is not a necessary condition for enhanced jet spreading. In fact, their direct numerical simulations indicate that the growth of swirled shear layers is augmented by a nonlinear interaction of a primary vortex ring and the columnar vortex that leads to a rapid breakdown of large flow structures into smaller scales.

Concerning the flow dynamics, it is widely accepted that the coexisting axial and azimuthal shear prevalent in swirling jets render the dominant vortical structures as highly three-dimensional. Panda \& McLaughlin (1994) successfully excited axisymmetric and non-axisymmetric modes in the shear layer of a turbulent swirling jet, revealing a lower receptivity for axisymmetric modes in comparison with the non-swirling jet. Billant, Chomaz \& Huerre (1998) and Loiseleux \& Chomaz (2003) investigated the dynamics in the nearfield of a natural laminar jet for various swirl intensities. They found large-scale co-winding double- and triple-helical structures in the pre-breakdown state that were perfectly steady for the experiments conducted by Billant et al. (1998), whereas Loiseleux \& Chomaz (2003) observed them to rotate at very low frequencies in the direction of the base flow rotation. In a previous study focusing mainly on post-breakdown conditions, it was shown that the swirling jet at pre-breakdown conditions is dominated by counter-rotating co-winding modes oscillating at much lower amplitudes than for the non-swirling case (Oberleithner et al. 2012). Gallaire \& Chomaz (2003b) conducted a temporal stability analysis of the mean profiles measured by Billant et al. (1998) showing that swirl destabilizes co-winding modes with high azimuthal wavenumbers due to the centrifugal instability in good agreement with the analysis of Leibovich \& Stewartson (1983). However, the most amplified azimuthal mode numbers disagree with the experimental observations of Billant et al. (1998), Loiseleux et al. (2000) and Oberleithner et al. (2012). Gallaire $\&$ Chomaz (2003b) attribute the discrepancy to the onset of a double-helical globally 
unstable mode with its wavemaker located at the nozzle lip. However, the authors admit that their arguments remain vague as they are based solely on the analysis at one streamwise location. The mode selection in the swirling jet experiments conducted by Liang \& Maxworthy (2005) differs significantly from the previously reported one. For various swirl intensities, all detected helical modes are co-rotating counter-winding and are aligned with the local helical vortex lines of the mean flow. The investigators argue that the relative location and thickness of the axial and azimuthal shear layers have a great influence on the streamwise evolution of the dominant modes. Hence, a comparison of their results with stability analysis based on simplified velocity models, as attempted by Liang \& Maxworthy (2005) remains only qualitative.

In this work we analyse the coherent structures that evolve in turbulent swirling jets at industry-relevant Reynolds numbers. The main questions addressed are: what are the dominant flow structures, what are their driving instabilities and how are they affected by swirl? As demonstrated by this brief and, by no means, comprehensive literature review, swirling jets are dominated by a large variety of flow instabilities that strongly depend on the particular underlying base flow. This hinders a consistent comparison of theoretical results typically based on simplified flow models and experimental observations. Therefore, we depart from model-based studies and apply linear stability analysis to time-averaged flows derived from experiments. The most unstable modes of the mean flow are then compared quantitatively to the measured coherent structures phase-locked by low-amplitude acoustic excitation. This approach implies two major assumptions: first, natural coherent structures are represented by linearly unstable modes of the underlying mean flow; second, the excited coherent structures behave equal to their natural equivalent. Among others, this approach was applied to the two-dimensional excited mixing layer (e.g. Gaster, Kit \& Wygnanski 1985) paving the way for efficient separation control (Greenblatt \& Wygnanski 2000), to the non-swirling jet (Cohen \& Wygnanski 1987a,b), and very recently to the swirling jet at post-breakdown conditions (Oberleithner et al. 2011).

In contrast to the investigation reported by Oberleithner et al. (2011), we here focus on swirl intensities below the onset of vortex breakdown where the flow is globally stable and the shear layers act as noise amplifiers. Owing to the absence of a single-tone instability, the mean flow analysis applies for a wide range of modes and, hence, covers a wide dynamical spectrum. The analysis is conducted for a range of swirl intensities revealing the explicit impact of swirl on the jet stability. We want to emphasize that there exists no universal swirling jet configuration and that the one investigated presently represents only one of several possibilities. Despite the loss of generality, which a generic flow model would probably provide, we are convinced that a detailed characterization of the instabilities of a particular flow configuration along with a quantitative experimental validation will enhance the general fundamental understanding, and it allows a proper interpretation of the findings obtained by other experimentalists.

The outline of the paper is as follows. The swirling jet facility, the measurement techniques, and the data treatment are outlined in $\S 2$. The four flow configurations considered in this study, ranging from zero swirl to strong swirl below the onset of vortex breakdown, are described in $\S 3$. Theoretical and numerical aspects concerning the spatial stability analysis applied to the time-averaged turbulent flow are described in $\S 4$. The numerically predicted instabilities at the nozzle exit are presented in $\S 5$, while their downstream development is described in $\S 6$. The results from the spatial analysis are compared with measurements conducted in the single-mode and pulsed 

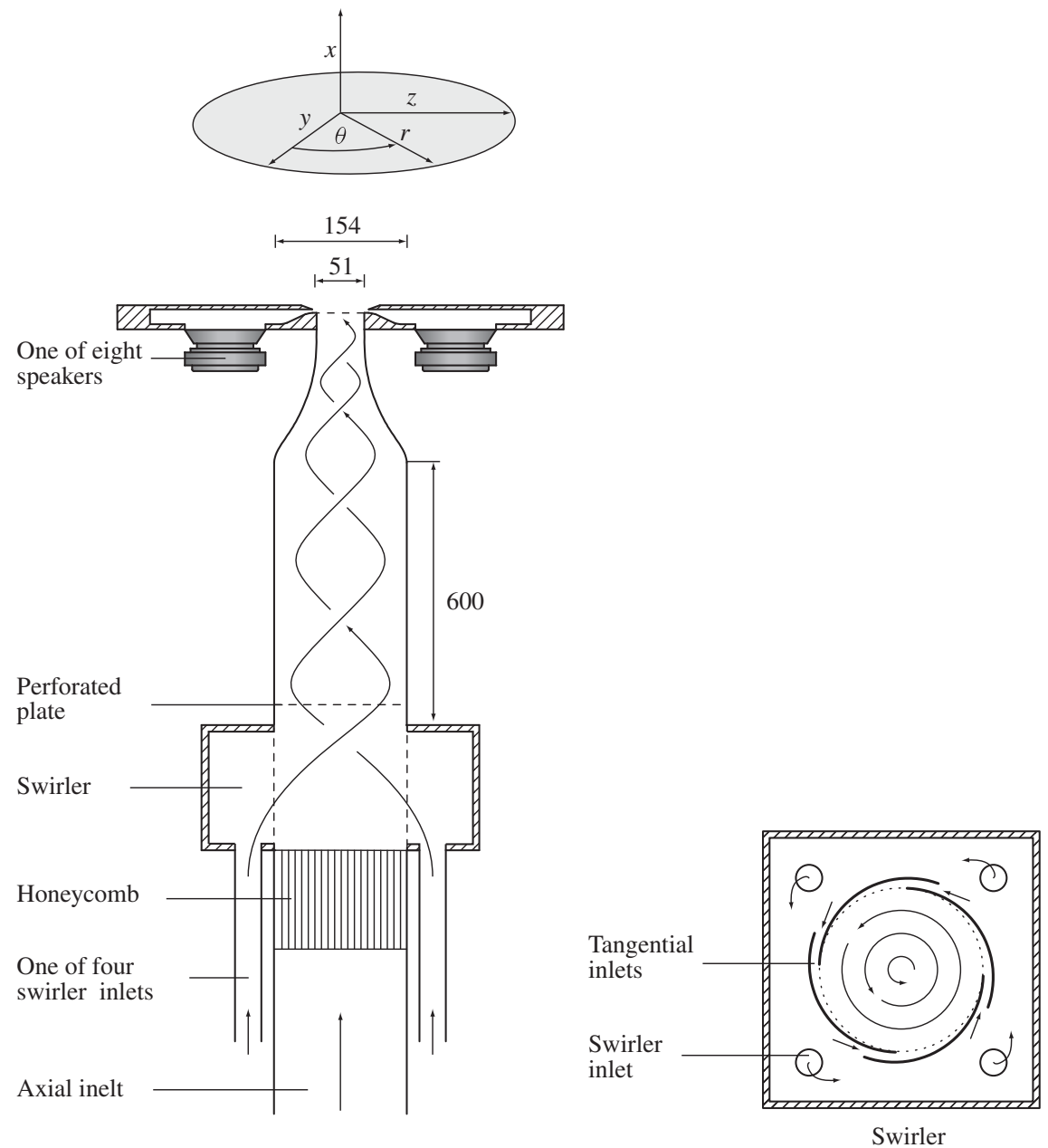

FIgURE 1. Experimental set-up and coordinate systems (all lengths are expressed in millimetres).

actuated shear layers in $\S \S 7$ and 8 , respectively. The major findings are summarized and discussed in $\S 9$.

\section{Experimental set-up and procedure}

\subsection{The swirling jet facility}

An unconfined turbulent swirling jet was generated by the same apparatus used in a previous investigation (Oberleithner et al. 2011). The schematic arrangement of the facility is shown in figure 1 together with the alignment of the coordinate system. Cylindrical coordinates are used throughout this work, with $x$ being originated at the nozzle exit, with the $x$-axis being aligned with the axis of rotation, with $r=0$ representing the jet centreline, and with $\theta$ pointing in positive direction according to the right-hand rule. The mean velocity components in the direction of the coordinates $(x, r, \theta)$ are $\left(V_{x}, V_{r}, V_{\theta}\right)$. Swirl was generated by mixing a primary axial air stream that passes through a deep honeycomb with a secondary air stream that enters the swirler 
through four tangential slots, each $80 \mathrm{~mm}$ long. The flow is then guided through a $600 \mathrm{~mm}$ long tube, before entering the contraction forming the nozzle. A perforated plate was mounted in the tube to minimize possible inhomogeneities resulting from the tangential inlets in the swirl chamber. The swirl levels generated by the facility depend on the ratio of mass flows coming through the two inlets: a non-swirling jet is generated when no air enters tangentially through the swirler and the maximum swirl level is attained when the axial inflow is zero. Two frequency-controlled blowers provided the necessary airflow. The volume flow of each blower was measured using calibrated orifices connected to BARATRON gauges. The blowers were feed-back controlled to provide a constant volume flux. The nozzle diameter is $D=51 \mathrm{~mm}$.

Fluidic excitation was applied using an array of eight loudspeakers equally spaced along the azimuth. Such an array provides radial fluctuations that trigger the shear-layer instabilities. An acoustic wave guide from each actuator terminates in a rectangular duct leading to a narrow slot that does not interfere with the jet flow when the speakers are inactive. The loudspeakers are driven by a set of digital-to-analogue converters under program control. The actuators were adjusted to equal amplitudes under no-flow conditions using a microphone located at the centreline in the exit plane of the nozzle. The azimuthal disturbances can be controlled by varying the phase difference between the actuators. With an array of eight actuators the highest azimuthal mode numbers that can be excited are $m= \pm 4$. A similar excitation device was successfully used by Long \& Petersen (1992) to study instabilities in non-swirling jets and by Panda \& McLaughlin (1994) for swirling jets. For the measurements of the impulse response, the flow was locally disturbed by using only one of the eight loudspeakers. A time and space discrete delta-pulse was generated by running the speaker with a saw-tooth signal with a sharp rising edge and a linearly decaying falling edge.

The choice of the actuation amplitude is not trivial as it has to be large enough to overcome the random turbulent noise in the shear layers. On the other hand, it must be sufficiently low to provide linear amplification. Moreover, forcing the flow at too high amplitudes would alter the mean flow substantially and thereby falsify the theoretical predictions that are based on the non-forced flow. The most appropriate amplitude for each flow configuration was derived through a preliminary parameter study. Therefore, the flow was forced at various amplitudes and a trade-off between good signal-to-noise level and a clear linear response was found.

\subsection{Measurement procedure}

Stereoscopic particle image velocimetry (Stereo-PIV) was used to measure the mean flow field and the corresponding turbulent shear stresses. This image-based method allows measurements of the velocity of particles going through a light sheet generated by a high-energy double-pulsed Nd:Yag laser at $532 \mathrm{~nm}$ and $160 \mathrm{~mJ}$. Two CCD cameras with a resolution of $2048 \times 2048$ pixels were used. Both cameras were positioned at a $45^{\circ}$ angle in back-scattering mode in order to measure all three velocity components in the $x-r$ plane. Each measurement ensemble consists of 900 snapshots captured at approximately $6 \mathrm{~Hz}$. The camera view angle allows measurements even inside the nozzle, which is necessary to have reliable data at the nozzle lip. The time delay between the two consecutive laser pulses was set according to the expected flow velocities to $50 \mu \mathrm{s}$. The strong out-of-plane velocity component associated with swirling flows required a rather short pulse separation in order to minimize lost particle pairs. 


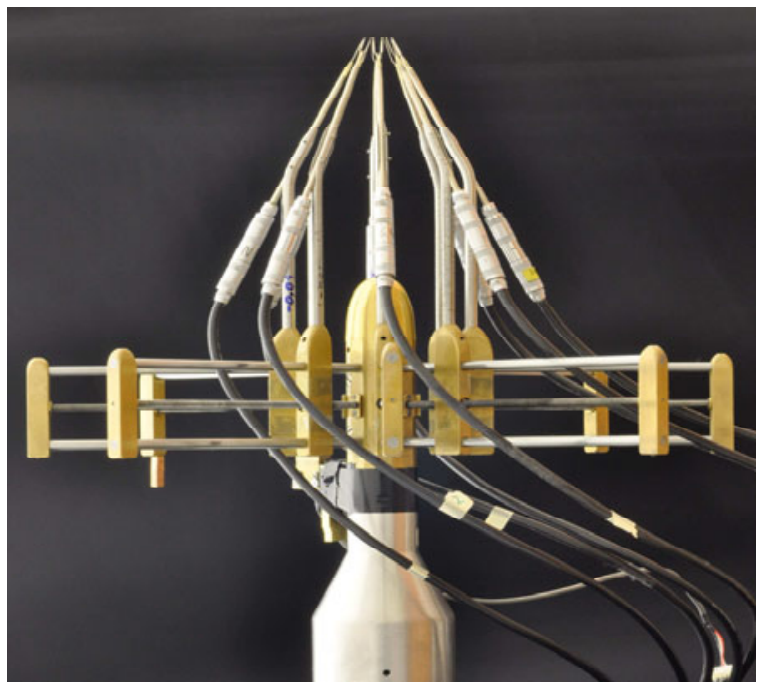

FIGURE 2. (Colour online) Hot-wire traversing system.

In order to detect the three-dimensional disturbance modes travelling along the shear layers, time-resolved volumetric measurements were conducted using eight hot-wire probes simultaneously. A unique traversing mechanism was used that simultaneously moves all hot-wires in radial and axial direction (figure 2). The hot-wire anemometers, which were built locally, were used in conjunction with a A.A.LAB SYSTEM LTD anemometer system. The hot-wires, made of tungsten, are $5 \mathrm{pm}$ in diameter. They were kept at a constant overheat ratio of 1.6 a maximum frequency response of $50 \mathrm{kHz}$. An analogue low-pass filter with a cut-off frequency of $6.2 \mathrm{kHz}$ was used to condition the measured signals prior to the analogue-to-digital converter. All channels were sampled simultaneously at $20 \mathrm{kHz}$, giving a maximum frequency response (Nyquist frequency) of $10 \mathrm{kHz}$.

The probes were distributed circumferentially around the jet centre, with the wires aligned parallel to the tangential velocity. This ensures the measurement of the axial velocity at a high spatial accuracy in the radial direction. The hot-wires were calibrated in the exit plane of the jet at no-swirl conditions against a standard Pitot tube at seven different velocities. Particular attention was paid to the radial adjustment of the hot-wire probes. This was done in no-swirl conditions by placing each of the eight hot-wires at the centre of the shear layer, where the mean velocity is reduced to one-half of the centreline value. After completing the fine alignment of the probes, all wires were traversed simultaneously in the radial and axial direction. Measurements were conducted at several axial and radial positions within the range $0<x / D<1.2$ and $0.2<r / D<0.8$.

In order to retain the phase information of the forced flow experiments, the excitation signal was recorded together with the signal from the hot-wire anemometer. In the case of sinusoidal excitation, the typical length of a velocity record used for averaging was equivalent to 1500 periods of the excitation frequency. In the case of pulsed-excited flow experiments, the record length was equivalent to 600 pulses, which were generated at $10 \mathrm{~Hz}$. 


$$
\begin{array}{lccc} 
& S_{1} & S_{3} & S_{4} \\
e_{\text {mean }}(\%) & 0.5 & 2.2 & 2.6 \\
e_{\text {rms }}(\%) & 3 & 3.3 & 2.8
\end{array}
$$

TABLE 1. Mean and r.m.s. error of the hot-wire measurements assuming PIV as the reference.

\subsection{Measurement uncertainty}

For the Stereo-PIV system, the measurement uncertainty strongly depends on the appropriate use of mapping functions along with suitable algorithms for recombination. A multigrid evaluation strategy was used (final interrogation size of $32 \times 32$ pixels at $50 \%$ overlap) including window deformation, Whittaker peak fitting and B-spline reconstruction. Errors due to misalignment of the laser sheet were minimized by the corrected mapping functions. Therefore, the datum marks for the initial calibration were back-projected onto the actual light-sheet plane via linear triangulation using the pinhole model. For this mapping and recombination algorithm, the measurement error is approximately 0.1 pixels (Raffel et al. 2007), which corresponds to an error for all three instantaneous velocity components of approximately $5 \%$ for the present arrangement. This error is randomly distributed, contributing mostly to the root-mean-square (r.m.s.) error. The presence of strong velocity gradients introduces an additional bias and r.m.s. error that depends on the interrogation size, the laser pulse delay and the seeding density. These errors are expected to be small due to the short time delays between the laser pulses used in the present experiments.

The main error sources for the single-probe hot-wire measurements are the interference of the traversing mechanism and probes with the flow and the threedimensionality of the flow at strong swirl. Figures 4 and 5 show mean velocity profiles measured with the hot-wires and with the PIV, revealing good overall agreement. The discrepancy between the two measurement techniques is quantified at the half-velocity radius $R_{.5}^{P I V}$, by using

$$
e=\left(\frac{V_{x}^{H W}-V_{x}^{P I V}}{V_{x}^{P I V}}\right)_{R_{5}^{P I V}},
$$

where the superscript indicates quantities derived from hot-wire or PIV data. Table 1 shows this quantity averaged over all streamwise measurement locations $e_{\text {mean }}$ and the corresponding r.m.s. deviation $e_{r m s}$. The mean error is increasing with increasing swirl, indicating a slight overestimation of the axial velocity with the addition of swirl. The r.m.s. error remains constant with increasing swirl at $\sim 3 \%$ revealing a satisfactorily small variation within the flow field.

\subsection{Basic data treatment}

For the single-mode actuated flow experiments, the coherent axial velocity component $v_{x}^{c}(\boldsymbol{x}, t)$ was derived from the phase-averaged hot-wire measurements. It is periodic in $t$ and $\theta$ and can be decomposed into a Fourier series with coefficients

$$
\hat{v}_{x}(x, r, m, n)=\frac{1}{2 \pi T} \int_{0}^{2 \pi} \int_{0}^{T} v_{x}^{c}(x, r, \theta, t) \mathrm{e}^{\mathrm{i}(m \theta-2 \pi n t / T)} \mathrm{d} \theta \mathrm{d} t .
$$

The coefficients $\hat{v}_{x}$ are complex, and $m$ and $n$ correspond to the azimuthal wavenumber and the time harmonic, respectively. Throughout this investigation, we focus only on 
the fundamental of the excited waves and set $n=1$. The amplitude of the forced mode at a given axial location $x$ is then derived by integrating the radial amplitude distribution across the axial shear layer (Delbende, Chomaz \& Huerre 1998),

$$
A_{m}(x)=\frac{1}{D}\left(\int_{R_{.05}}^{R_{.95}}\left|\hat{v}_{x}\right|^{2} r \mathrm{~d} r\right)^{1 / 2}
$$

with $R_{0.95}$ and $R_{0.05}$ corresponding to the radial positions where the mean axial velocity is 95 and $5 \%$ of the bulk velocity $V$, respectively. The corresponding phase distribution is

$$
\varphi_{m}(x, r)=\arg \left(\hat{v}_{x}\right) .
$$

The pulsed-excited experiments were conducted in order to capture a single wave packet travelling along the swirling jet shear layer. In order to reduce noise caused by turbulent fluctuations, the experiments were repeated 600 times and were ensemble-averaged. A detailed description of this method is given by Gaster \& Grant (1975). The method was further finalized to reduce the ambiguity in the averaged quantities due to jitter in the arrival time and location. These irregularities, which are induced by random turbulent motion of the base flow, may blur the actual shape of the wave packet. Therefore, the signal of each individual recorded wave packet, which is a function of $\theta$ and $t$, was cross-correlated with the ensemble-averaged signal. The correlation peak provides an estimate of the $t$ - and $\theta$-displacement of each pulse with respect to the mean values. The displacement was then compensated for each pulse and the ensemble average was redone. This procedure was repeated iteratively until convergence was achieved. For a detailed description of this method, the reader is referred to Zhou, Heine \& Wygnanski (1996) and references therein.

\section{Characterization of the base flows}

The mean flow in the $x-r$ plane was derived from the Stereo-PIV measurements for different swirl intensities at a constant Reynolds number of $R e_{D}=D V_{0} / v=20000$, with $V_{0}$ being the bulk velocity at the nozzle and $v$ the kinematic viscosity of air.

\subsection{Analytic representation of the mean flow}

The measured mean flow is approximated by profiles given by the following analytic expressions:

$$
\begin{gathered}
V_{x}=\frac{V}{1+\left(\exp \left[\left(r / R_{.5}\right)^{2} \log (2)\right]-1\right)^{N_{x}}}, \\
V_{r}=0, \\
V_{\theta}=\Omega_{c l} r \exp \left[-\left(r / R_{\theta}\right)^{N_{\theta}} / N_{\theta}\right] .
\end{gathered}
$$

A slightly modified version of the expression for the axial velocity component $V_{x}$ was introduced by Monkewitz \& Sohn (1988). Later, Gallaire \& Chomaz (2003b) used this profile to approximate the measurements conducted by Billant et al. (1998). Here $V$ represents the bulk velocity and $R_{.5}$ the jet radius, defined as the radial distance where $V_{x}=0.5 \mathrm{~V}$. The dimensionless parameter $N_{x}$ is related to the radial gradient of the axial velocity profile and is inversely proportional to the axial shear-layer thickness. The model for the azimuthal velocity component $V_{\theta}$ was first introduced by Carton \& McWilliams (1989) and more recently used by Gallaire \& Chomaz (2003b). We use $\Omega_{c l}$ to represent the rotation rate on the jet axis and $R_{\theta}$ to represent 

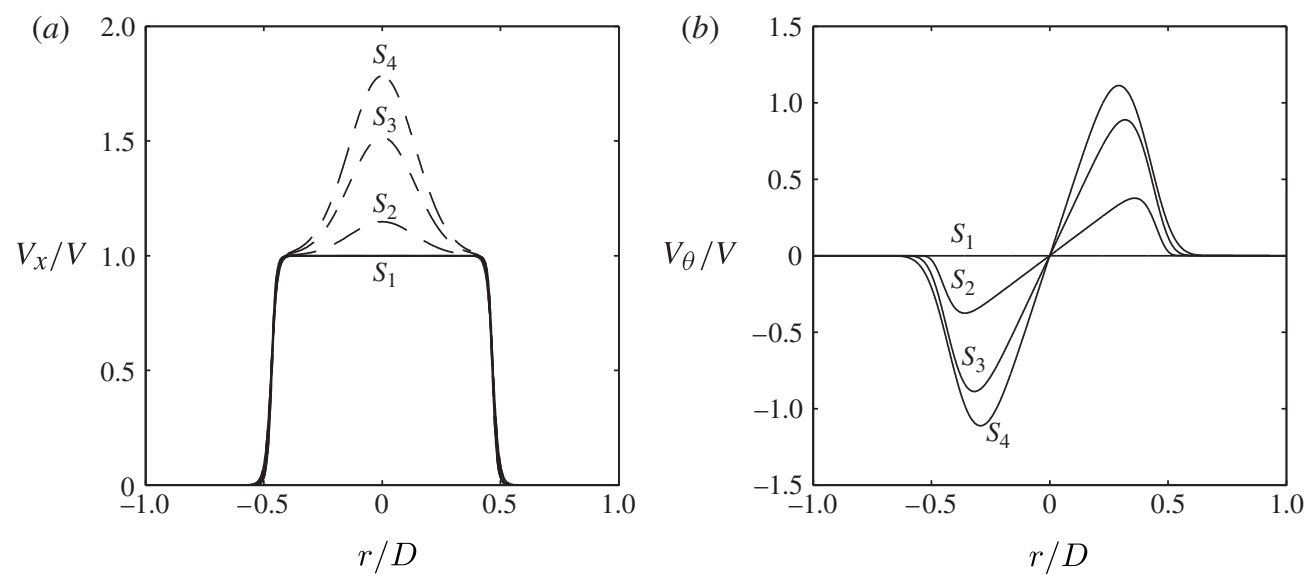

FIGURE 3. Mean velocity profiles at the nozzle for different swirl intensities. The solid lines correspond to the simple model (3.1) and the dashed lines correspond to model (3.2) that compensates for the axial overshoot. The model parameters are given in table 2 .

the radial location of the maximum azimuthal velocity. The dimensionless parameter $N_{\theta}$ is inversely proportional to the azimuthal shear-layer thickness. The parameters $N_{x}$ and $N_{\theta}$ are determined from a least-squares fit of the expressions (3.1a) and (3.1c) to the axial and azimuthal mean velocity, respectively.

For the swirling jet, the contraction upstream of the nozzle generates an overshoot of the axial velocity profile in agreement with previous studies (Panda \& McLaughlin 1994; Billant et al. 1998; Semaan, Naughton \& Ewing 2009). To account for this hump in the axial velocity profile, Gallaire \& Chomaz (2003b) introduced an additional term to $(3.1 a)$, yielding the more complex expression

$$
V_{x}=\frac{V}{1+\left(\exp \left[\left(r / R_{.5}\right)^{2} \log (2)\right]-1\right)^{N_{x}}}+V_{o s} \exp \left[-\left(r / r_{o s}\right)^{2}\right],
$$

where $V_{o s}$ represents the strength of the overshoot and $r_{o s}$ its radial extent. According to Gallaire \& Chomaz (2003b), the axial overshoot has minor effect on the instability of the base flow. Preliminary computations conducted for both models confirm the insignificance of the overshoot for the present flow and, therefore, the stability analysis presented throughout this study is based on the mean flow approximated by the simpler and far more generic model (3.1).

\subsection{The velocity distribution at the nozzle}

The back-scattering configuration of the PIV measurements enables access to the flow quantities at the nozzle exit. The fitted profiles of the axial and azimuthal mean velocities are shown in figure 3. Measurements were conducted at four different swirl configurations ranging from zero swirl, labelled by $S_{1}$, to strong swirl, labelled by $S_{4}$. The solid lines correspond to the simple model (3.1) and the dashed lines correspond to model (3.2) that compensates for the axial overshoot. The model parameters are given in table 2 .

Despite the formation of an overshoot, the swirl does not significantly alter the axial velocity profile, which is in agreement with measurements of laminar swirling jets 


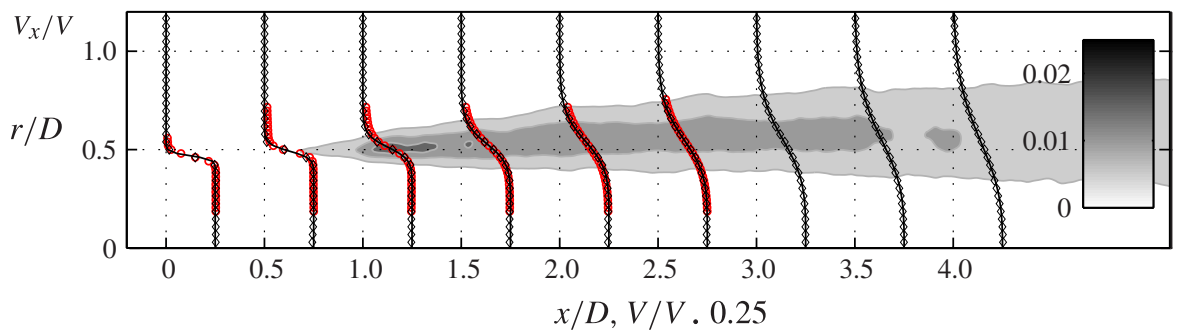

FIGURE 4. (Colour online) Non-swirling jet configuration $S_{1}$ : profiles of the axial velocity are shown together with contours of the turbulent shear stress $\overline{v_{x}^{\prime} v_{r}^{\prime}} / V^{2}$. Diamond-shaped symbols refer to PIV measurements, hollow circles (shown in red online) to hot-wire measurements and solid black lines refer to the analytic approximation $(3.1 a)$.

$\begin{array}{lcccc} & S_{1} & S_{2} & S_{3} & S_{4} \\ V / V_{0} & 1 & 1 & 1 & 1 \\ \Omega_{c l} R_{.5} / V & 0 & 0.54 & 1.54 & 2.23 \\ R_{.5} / D & 0.47 & 0.47 & 0.47 & 0.47 \\ R_{\theta} / D & - & 0.36 & 0.32 & 0.29 \\ N_{x} & 17.78 & 16.74 & 14.61 & 12.75 \\ N_{\theta} & - & 10.37 & 6.14 & 4.45 \\ r_{o s} & - & 0.42 & 0.42 & 0.42 \\ V_{o s} / V & - & 0.15 & 0.52 & 0.78\end{array}$

TABLE 2. Parameters of the mean flow approximation (3.1) determined by a least-squares fit to the measured velocity profiles at at $x / D=0$.

(Billant et al. 1998). The axial shear-layer thickness increases slightly with increasing swirl, indicated by a decrease of $N_{x}$. The azimuthal velocity profile is certainly affected by an increase in swirl. The rotation rate near the jet axis, expressed as the dimensionless ratio $\Omega_{c l} R_{.5} / V$, reaches 2.23 at highest swirl. Moreover, the radial position of the maximum azimuthal velocity $R_{\theta}$ moves towards the jet centre and the azimuthal shear layer thickens considerably, indicated by a decrease of $N_{\theta}$. The latter is not observed in the laminar swirling jet experiments conducted by Billant et al. (1998) and Loiseleux \& Chomaz (2003), where $N_{\theta}$ and $R_{\theta}$ remain constant with increasing swirl.

\subsection{Quantification of swirl at the nozzle exit}

Several swirl number definitions exist in the literature, each being applicable to a certain flow regime, but none being universal. Table 3 lists the most common swirl numbers derived at the nozzle exit for the four flow configurations considered. Throughout this work, they will be simply referred to as $S_{1}, S_{2}, S_{3}$ and $S_{4}$. A comparison with the listed literature reveals that the highest swirl number $S_{4}$ is still below the critical level where vortex breakdown occurs.

\subsection{Streamwise distribution of mean velocities and turbulent stresses}

Figures 4 and 5 depict the mean flow distribution along the streamwise plane of measurement derived from PIV measurements for the non-swirling and swirling jet, 
(a)

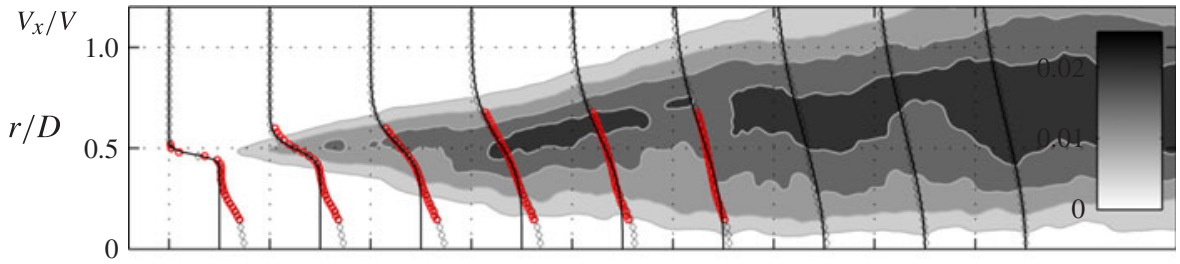

(b)

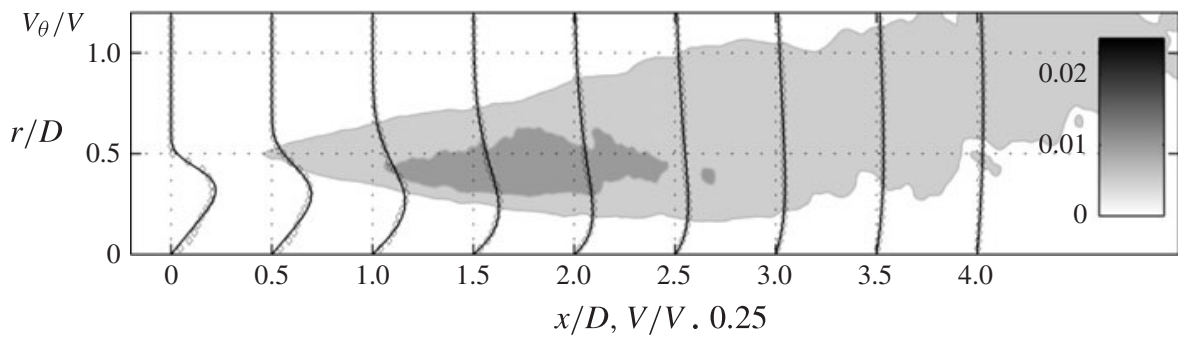

FIgURE 5. (Colour online) Swirling jet configuration $S_{3}$ : profiles of the axial $(a)$ and the azimuthal $(b)$ velocity component are shown together with contours of the turbulent shear stress $\overline{v_{x}^{\prime} v_{r}^{\prime}} / V^{2}$ and $\overline{v_{r}^{\prime} v_{\theta}^{\prime}} / V^{2}$, respectively; diamond-shaped symbols refer to PIV measurements, hollow circles (shown in red online) to hot-wire measurements and solid black lines refer to the analytic approximation (3.1).

$\begin{array}{lccccc} & S_{1} & S_{2} & S_{3} & S_{4} & \text { Critical threshold } \\ \frac{2 \int_{0}^{\infty} V_{x} V_{\theta} r^{2} \mathrm{~d} r}{D \int_{0}^{\infty}\left(V_{x}^{2}-V_{\theta}^{2} / 2\right) r \mathrm{~d} r} & 0 & 0.19 & 0.46 & 0.6 & 1.06 \text { (Oberleithner } \text { et al. 2012) } \\ \frac{\sqrt{\int_{0}^{\infty} V_{\theta}^{2} / r \mathrm{~d} r}}{V_{x}(r=0)} & 0 & 0.28 & 0.55 & 0.62 & 0.71 \text { (Billant } \text { et al. 1998) } \\ \frac{2 V_{\theta, \max }}{V_{x, \max }} & 0 & 0.66 & 1.17 & 1.25 & 1.41 \text { (Escudier \& Keller 1983) } \\ \frac{2 V_{\theta}(r=D / 4)}{V_{x}(r=0)} & 0 & 0.5 & 1.04 & 1.19 & 1.30 \text { (Billant } \text { et al. 1998) } \\ \left(\frac{\Omega_{c l} R_{\theta}}{V}\right)^{-1} & \infty & 2.41 & 0.96 & 0.72 & 0.69 \text { (Liang \& Maxworthy 2005) } \\ \arctan \left(\frac{V_{\theta}}{V_{x}}\right)_{\max } & 0 & 20.52 & 40.64 & 46.01 & 50.0 \text { (Sarpkaya 1971) }\end{array}$

TABLE 3. Different swirl number definitions evaluated at $x=0$ and corresponding critical thresholds for vortex breakdown taken from previous investigations.

respectively, together with the velocity profiles approximated by the simplified model (3.1). The agreement between the fit and the actually measured values is excellent for the non-swirling jet. For swirl configuration $S_{3}$, the analytic approximation of the axial velocity component is also good, despite a slight underestimated shear-layer thickness 
near the nozzle exit. The swirl profiles are represented reasonably well by the model, however, upstream of $x / D=1$ the inflection point inside the vortex core region is not reproduced. For comparison reasons, the axial velocity profiles derived from the hot-wire measurements are also shown for the swirling and non-swirling jet. Despite the overestimation observable in the outer jet region, the profiles obtained from the hot-wire measurements are nearly indistinguishable from the PIV data.

The contour surfaces shown in figures 4 and 5 indicate regions of high turbulent production. They represent the normalized turbulent shear stress component $\overline{v_{x}^{\prime} v_{r}^{\prime}}$ for the non-swirling jet, and $\overline{v_{x}^{\prime} v_{r}^{\prime}}$ and $\overline{v_{r}^{\prime} v_{\theta}^{\prime}}$ for the swirling jet. Without swirl, $\overline{v_{r}^{\prime} v_{\theta}^{\prime}}$ is orders of magnitude smaller than for the swirling case and is therefore covered in noise (not shown). The contours reveal that the radial maximum is located approximately at the highest radial gradient of $V_{\theta}$, indicating that $\overline{v_{r}^{\prime} v_{\theta}^{\prime}}$ is primarily generated by the azimuthal shear. The turbulent shear stress component $\overline{v_{x}^{\prime} v_{r}^{\prime}}$ is also enhanced by the addition of swirl, which presumably results in an enhanced downstream growth of the axial shear layer and a shortening of the potential core region. Furthermore, the turbulent shear stresses confirm that the axial overshoot near the nozzle, which creates an additional inflection point in the axial velocity profile, does not contribute significantly to the turbulent production and, hence, instabilities growing in the inner shear layer seem to be negligible. Similarly, the aforementioned inflection point of the azimuthal profile near the jet centre upstream of $x / D=1$ does not generate turbulent shear stress and it is not expected to promote dominant instabilities. The poor approximation of this feature is therefore considered to be insignificant. Note that PIV measurements are only conducted for the unforced flow where the coherent velocity cannot be extracted. Hence, the turbulent shear stresses discussed here are derived from the fluctuating velocity field that comprises stochastic and coherent motion.

\subsection{Parametrization of the mean flow}

The evolution of the mean flow with downstream distance is characterized by the streamwise distribution of the parameters of model (3.1). The bulk velocity $V$ is displayed in figure $6(a)$. It is taken as the characteristic length scale throughout this work. The other parameters that scale the axial velocity profiles are shown in figure $6(c, e)$ and those corresponding to the azimuthal velocity profiles are shown in figure $6(b, d, f)$. Figure $6(g)$ displays the streamwise development of the momentum thickness $\delta_{x}$. It is used as the characteristic length scale throughout this work and represents a measure for the axial shear layer thickness, defined as

$$
\delta_{x}=\int_{R_{.05}}^{R_{.95}} \frac{V_{x}}{V}\left(1-\frac{V_{x}}{V}\right) \mathrm{d} r .
$$

Figure $6(a)$ indicates the axial extent of the potential core, the region where $V$ is equal to the bulk velocity at the nozzle $V_{0}$. For the non-swirling jet $\left(S_{1}\right)$, the potential core exceeds the measurement domain. By increasing the swirl, the potential core region is shortened significantly due to the increased growth of axial shear-layer thickness. For $S_{3}, V$ declines for $x / D>2$, whereas Loiseleux \& Chomaz (2003) measures a potential core length of $x / D \approx 3.8$ at a comparable swirl intensity. Hence, the presently found streamwise extent of the potential core is much smaller than for laminar swirling jets.

It is interesting to note that the swirl parameter (inverse Rossby number) shown in figure $6(b)$ remains perfectly constant within the potential core, despite the downstream decay of the maximum swirl velocity $V_{\theta, \max }$. It is the only local swirl 

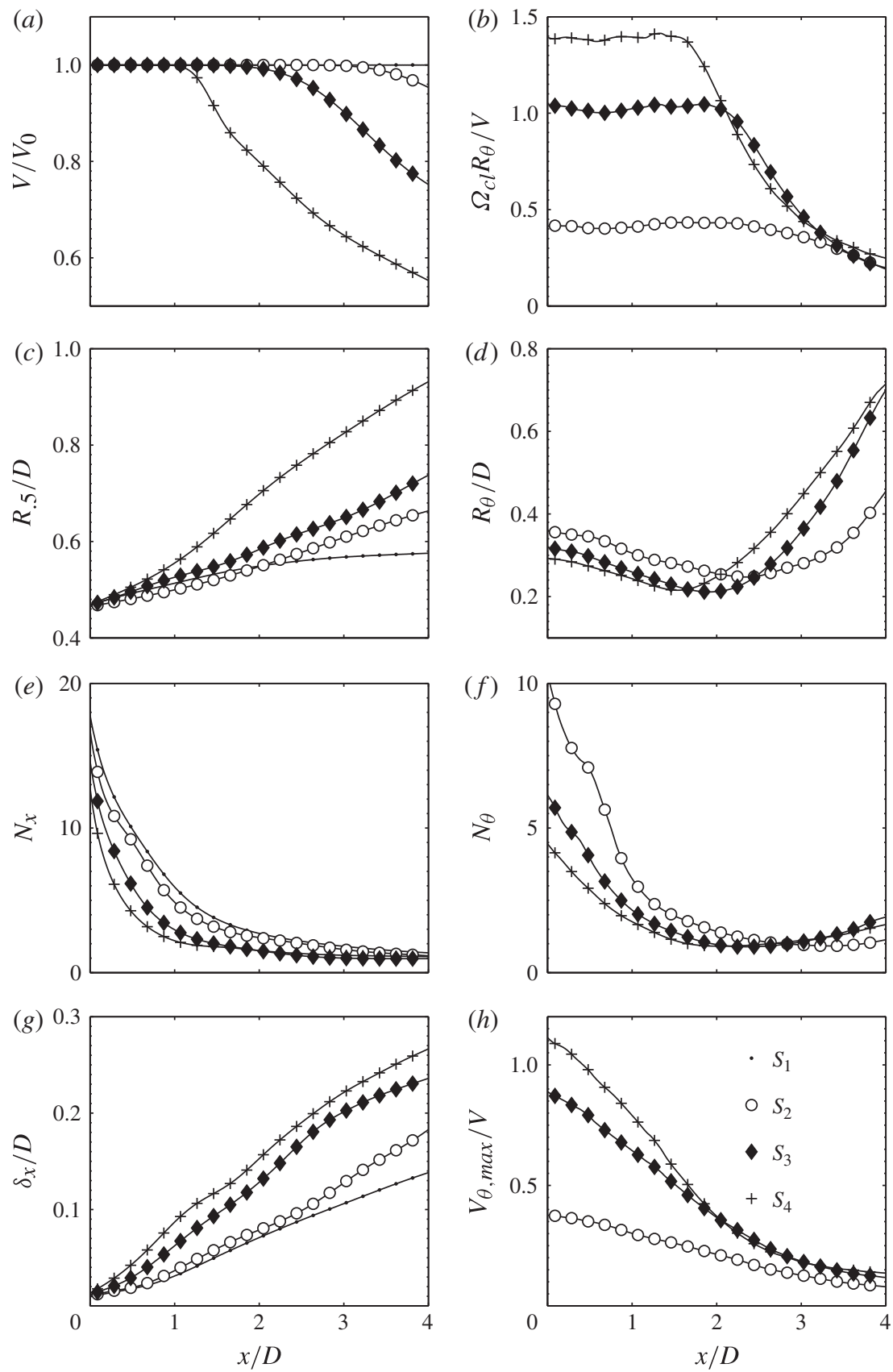

FIgURE 6. $(a-f)$ Streamwise development of mean flow parameters of (3.1); Streamwise development of axial momentum thickness $(g)$ and maximum swirl velocity $(h)$.

number of the definitions listed in table 3 that remains constant within the potential core. It is further worth noting that downstream of the potential core, the Rossby number decays at the same rate independent of the swirl intensity and it ultimately collapses to one curve that asymptotes zero. 
The quantities shown in figure $6(c, e$ and $f)$ indicate the enhanced jet spreading with increasing swirl. The non-monotonic development of $R_{\theta}$ is somewhat unexpected (figure $6 d$ ). Within the potential core, the downstream growth of the azimuthal shear layer leads to a displacement of the maximum of the swirl component to the jet centre, while the constant swirl parameter (figure $6 b$ ) implies that $\Omega_{c l}$ must increase correspondingly. Downstream of the potential core, the maximum of $V_{\theta}$ moves outwards and the azimuthal shear-layer thickness decreases, indicated by an increasing shape parameter $N_{\theta}$. The maximum azimuthal velocity $V_{\theta \text {,max }}$ decelerates drastically within the potential core and collapses for all swirl configurations at $x / D>3.5$ in a similar manner as the inverse Rossby number. Apparently, the growth of the azimuthal shear layer is coupled to the growth of the axial shear layer in the potential core region, which leads to a constriction of the vortex core. Further downstream, the axial shear layer merges on the jet axis, the vortex core spreads radially and the inverse Rossby number decays.

\section{Implementation of the linear stability analysis}

\subsection{Theoretical concept}

Linear stability analysis is commonly applied to predict the initial growth of small perturbations travelling on a stationary laminar base flow. Once the perturbation reaches a sufficient size, nonlinearities set in and the linear analysis is biased. This type of analysis is mostly applied to computed base flow models providing useful insights into the transition to turbulence.

In order to model perturbations growing in turbulent flows, the laminar flow models are less representative and it is more appropriate to apply the analysis on the time-averaged flow. This procedure renders the analysis inherently nonlinear due to the turbulent Reynolds stresses that form the mean flow. Substituting the turbulent mean flow for the stationary laminar flow violates the assumptions made to derived the instability equations. Therefore, the analysis must not be generally valid and a cross-check with experiments is required. Although, this approach is mathematically less rigourous, its outcome reflects experimental findings in a more consistent manner. The success of this semi-empirical approach was demonstrated by various investigations, revealing that linear stability analysis is a powerful tool to model large-scale coherent structures in turbulent flows. Accordingly, this method can be applied at different degrees of complexity and accuracy, ranging from local stability analysis (Gaster et al. 1985; Cohen \& Wygnanski 1987a; Pier 2002; Juniper, Tammisola \& Lundell 2011; Oberleithner et al. 2011) through parabolized stability analysis (Herbert 1997; Gudmundsson \& Colonius 2011) to global stability analysis (Barkley 2006; Meliga, Pujals \& Éric 2012).

Instability wave models in turbulent flows are typically based on a separation of scales which is formalized by the classical triple decomposition introduced by Hussain \& Reynolds (1970),

$$
\boldsymbol{v}(\boldsymbol{x}, t)=\boldsymbol{V}(\boldsymbol{x})+\boldsymbol{v}^{c}(\boldsymbol{x}, t)+\boldsymbol{v}^{s}(x, t) .
$$

Accordingly, the time- and space-dependent flow $\boldsymbol{v}(\boldsymbol{x}, t)$ is decomposed into a time-averaged part $\boldsymbol{V}(\boldsymbol{x})$, a coherent part $\boldsymbol{v}^{c}(\boldsymbol{x}, t)$ and a randomly fluctuating (stochastic) part $\boldsymbol{v}^{s}(\boldsymbol{x}, t)$. The mean part interacts with the coherent part through a mean flow correction that is induced by coherent Reynolds stresses generated by the spatially growing/decaying coherent structures (Reau \& Tumin 2002; Noack et al. 2003). The fine-scale turbulence acts on the coherent part by adding a fictitious eddy 
viscosity reducing the growth rate of the inherent instability. Taking this into account, the eigenmodes of the mean flow reflect an equilibrium state between spatially growing/decaying flow structures and corresponding mean flow corrections. This allows the modelling of the coherent structures beyond their initial growth, resolving their saturation and partly their decay.

\subsection{The local linear stability equations}

The linear stability analysis conducted in this work is in line with a previous investigation dealing with the coherent structures in strongly swirling jets undergoing vortex breakdown. Therefore, the mathematical concept is only briefly introduced. For a comprehensive representation of the governing equations and the validation of the numerical procedure, the reader is referred to Oberleithner et al. (2011).

Following the procedure of local linear stability analysis, the velocity and pressure disturbances, represented by the coherent components $\left(v_{x}^{c}, v_{r}^{c}, v_{\theta}^{c}, p^{c}\right)$, are superposed onto the measured mean velocity profile. The perturbations that are periodic in time, in the streamwise and in the azimuthal direction, are decomposed into normal modes, yielding

$$
\begin{gathered}
\boldsymbol{v}^{c}(x, r, \theta, t)=\hat{\boldsymbol{v}}(r) \mathrm{e}^{\mathrm{i}(\alpha x+m \theta-\omega t)}+\hat{\boldsymbol{v}}^{*}(r) \mathrm{e}^{-\mathrm{i}\left(\alpha^{*} x+m \theta-\omega^{*} t\right)} \\
p^{c}(x, r, \theta, t)=\hat{p}(r) \mathrm{e}^{\mathrm{i}(\alpha x+m \theta-\omega t)}+\hat{p}^{*}(r) \mathrm{e}^{-\mathrm{i}\left(\alpha^{*} x+m \theta-\omega^{*} t\right)},
\end{gathered}
$$

where $\alpha$ is the complex streamwise wavenumber, $\omega$ the complex frequency and $m$ the real integer azimuthal wavenumber. Upon substituting (4.2) into the Navier-Stokes equations, linearized about the mean flow $\boldsymbol{V}(x, r, \theta)$, we obtain

$$
\nabla \cdot v^{c}=0
$$

for the continuity equation and

$$
\frac{\partial \boldsymbol{v}^{c}}{\partial t}+\boldsymbol{v}^{c} \cdot \boldsymbol{\nabla} \boldsymbol{V}+\boldsymbol{V} \cdot \boldsymbol{\nabla} \boldsymbol{v}^{c}=-\nabla p^{c}+\frac{1}{R e} \nabla^{2} \boldsymbol{v}^{c}
$$

for the momentum equation.

Equipped with appropriate boundary conditions (see Oberleithner et al. 2011), the system (4.4) can be written as an eigenvalue problem with either $\alpha$ or $\omega$ being the eigenvalue and $(\hat{\boldsymbol{v}}, \hat{p})$ the eigenfunctions.

\subsection{Analysis of divergent flows: a quasi-parallel approach}

The above-introduced eigenvalue problem is solved for a complex streamwise wavenumber $\alpha=\alpha_{r}+i \alpha_{i}$ and a given real frequency $\omega_{r}$. This approach is called spatial analysis, yielding spatially growing $\left(-\alpha_{i}>0\right)$ or decaying $\left(-\alpha_{i}<0\right)$ modes. It is applicable to open shear flows that are convectively unstable (noise amplifiers, see Michalke 1965). In contrast, absolutely unstable flows featuring self-excited instabilities (flow oscillators, see Huerre \& Monkewitz 1990) are typically treated with a spatiotemporal analysis solving the eigenvalue problem for complex streamwise wavenumbers and frequencies. In a preliminary study, it was assured that the present flow is not absolutely unstable for $|m| \leqslant 3$, justifying the approach adopted here. The spatial analysis describes the streamwise growth and decay of flow perturbations initiated at a certain axial location, e.g. $x=0$. Within the framework of the multiple-scale analysis, the global disturbance velocity field can be reconstructed from the local analysis using

$$
\boldsymbol{v}^{c}(\boldsymbol{x}, t ; \epsilon)=\Re\left\{A_{0}(x) \hat{\boldsymbol{v}}(x, r ; \epsilon) \exp \left[\frac{\mathrm{i}}{\epsilon}\left(\int_{0}^{x} \alpha(\xi) \mathrm{d} \xi+m \theta-\omega_{r} t\right)\right]\right\},
$$


where the eigenvalue $\alpha$ and the eigenfunction $\hat{\boldsymbol{v}}$ is derived at each streamwise slice separately. The parameter $\epsilon$ quantifies the streamwise non-uniformity of the flow. It must be small for the local analysis to be rigourously valid, implying a slow variation of the eigenfunction with $x$ and a fast variation of the wavenumber $\alpha$ with $\xi$. Assuming that the rate of divergence is characterized by the momentum thickness of the axial shear layer $\delta_{x}$, we find $\epsilon=\mathrm{d} \delta_{x} / \mathrm{d} x<0.07$ for all considered configurations, which justifies the weakly non-parallel approximation.

Crighton \& Gaster (1976) have developed a first-order correction for weakly non-parallel flows that enables to derive a slowly varying amplitude scaling $A_{0}(x)$ from a ordinary differential equation employing the eigenfunction and its adjoint. By accounting for the term $A_{0} \hat{\boldsymbol{v}}$ the growth rate and phase velocity of a travelling wave becomes dependent on the streamwise and radial coordinate and on the velocity component. For the sake of simplicity, we omit the weakly non-parallel correction and assume $A_{0}$ in (4.5) to be uniform and the eigenfunction $\hat{\boldsymbol{v}}(x, r)$ to depend parametrically on $x$. The latter are arbitrarily normalized with respect to the Euclidean norm $\|\hat{\boldsymbol{v}}\|=\sqrt{(\hat{\boldsymbol{v}}, \hat{\boldsymbol{v}})}$, which introduces an ambiguity to the present analysis, however noting that the normalization with other quantities such as the total kinetic energy or the radially integrated amplitude did not noticeably affect the results. The quasi-parallel approach adopted here provides a coarse but comprehensive picture of the instabilities that dominate this flow and how they are affected by swirl. As a drawback, this simple approach is incapable of revealing higher-order intermodal interactions that may be present due to the non-parallelism of the flow.

\subsection{Turbulence model}

The impact of turbulence on the growth of the coherent structures is modelled assuming that fine-scale turbulence provides additional mixing and behaves like an added eddy viscosity. The instabilities in free-shear flows are typically inviscid and, hence, viscosity has primarily a stabilizing effect (see e.g. Liu 1971; Marasli, Champagne \& Wygnanski 1991; Reau \& Tumin 2002). The eddy viscosity $v_{t}$ adds to the kinematic viscosity $v$, significantly reducing the Reynolds number used in the stability equations (4.4).

The eddy viscosity is derived from the experimental data employing the well-known Boussinesq approximation, utilizing the expression $-\overline{v_{x}^{\prime} v_{r}^{\prime}}=v_{t}\left(\partial V_{x} / \partial r+\partial V_{r} / \partial x\right)$. As stated by Townsend (1956), this simple eddy viscosity model is only valid within the turbulent flow and must therefore be weighted by an intermittency factor $\gamma$ that is derived from the PIV snapshots using the following approach: first, the instantaneous azimuthal vorticity is calculated from each PIV snapshot; second, the noise from the obtained vorticity field is removed by setting values below $5 \%$ to zero. The resulting vorticity distributions provide a reasonably good description of the instantaneous boundary between rotational and irrotational flow. Third, the number of events of irrotational flow $N_{\text {pot }}$ are derived for each measurement location. The intermittency function is then defined as $\gamma=N_{p o t} / N$, where $N$ is the total number of PIV snapshots.

The eddy viscosity is weighted by the intermittency factor and averaged in the radial direction. It is displayed in figure $7(a)$. For all swirl configurations, $v_{t}$ is nearly equal at the nozzle exit and grows rapidly with downstream distance. The growth is enhanced with increasing swirl in consistency with the enhanced shear-layer growth discussed in $\S 3.5$. Accounting for the added turbulent viscosity, the Reynolds number 

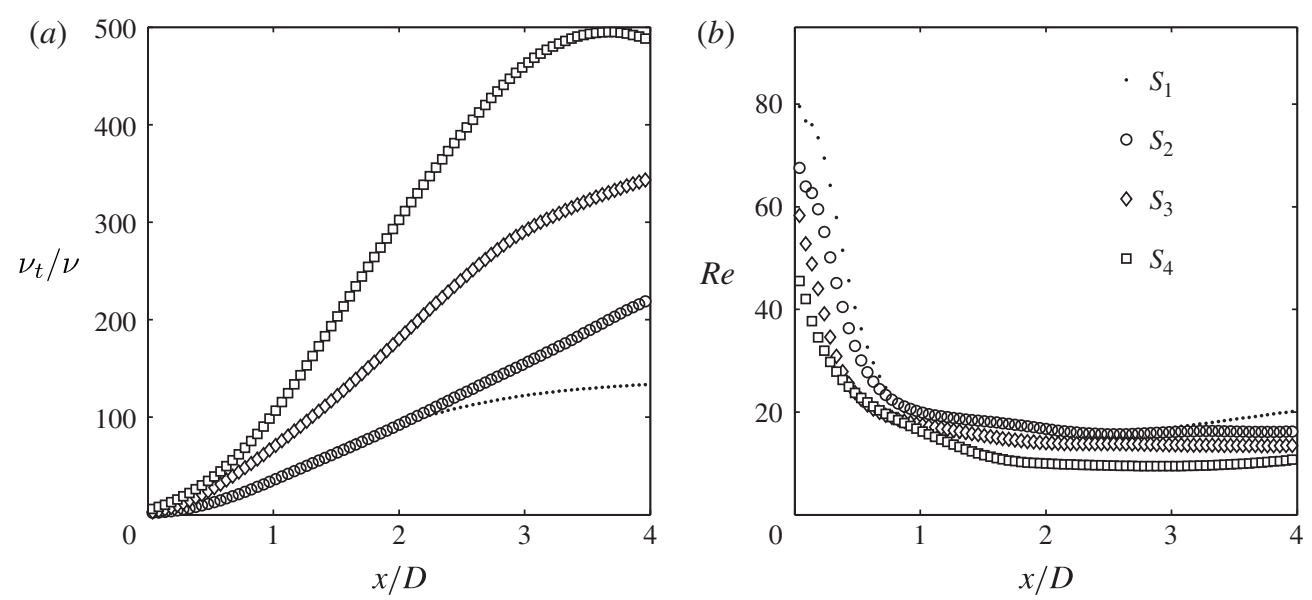

FIgURE 7. (a) Streamwise distribution of eddy viscosity $v_{t}$ derived from PIV measurements and $(b)$ Reynolds number $\operatorname{Re}=\left(V \delta_{x}\right) /\left(v+v_{t}\right)$ used for the stability analysis.

in the stability equations (4.4) is now defined as

$$
R e=\frac{V \delta_{x}}{v+v_{t}} .
$$

It is displayed in figure $7(b)$. For all swirl configurations, it decays rapidly upstream of $x / D=1$ and then asymptotes at similar constant values within the range $10<R e<20$. The growth of eddy viscosity appears to be balanced by the growth of momentum thickness.

\subsection{Numerical implementation}

The local linear stability eigenvalue problem is efficiently solved by using a Chebyshev spectral collocation method (Khorrami, Malik \& Ash 1989; Trefethen 2000). A detailed description of the numerical scheme is given by Oberleithner et al. (2011). It is further simplified for the present study by implementing the MATLAB $^{\mathrm{TM}}$ routine EIGS, which solves an eigenvalue problem and returns only one eigenvalue that is closest to a given estimate. The eigenvalue problem is first solved for one parameter combination by using the standard MATLAB ${ }^{\mathrm{TM}}$ routine EIG, which returns numerous eigenvalues including the spurious ones. The physically meaningful solutions are then sorted out, a posteriori, by using two independent criteria as mentioned by Oberleithner et al. (2011). Starting from this solution, the eigenvalue problem is solved for a new parameter combination using the EIGS routine. The required estimate is thereby derived from cubic extrapolation of the already obtained solutions while going in small increments through the parameter space. Particular attention must be paid when several modes exist for one parameter setting since the EIGS routine may switch from one mode to the other. All results are cross-checked by using the standard EIG routine. The computations are conducted using 200 Chebyshev points. A two-parameter transformation proposed by Malik, Zang \& Hussaini (1985) is used to map the Chebyshev collocation points onto the physical domain of the problem, in line with the previous study (Oberleithner et al. 2011). The boundary conditions for $r=\infty$ are applied at $r=100 D / \delta_{x}$ to account for the spatially varying length scale. 


\section{Instabilities at the nozzle exit}

At the nozzle exit, the top-hat axial velocity profile is nearly independent of the swirl, while the azimuthal component, characterized by a linear region near the jet centre (solid-body rotation) and an outward decaying region (azimuthal shear layer), depends strongly on the swirl intensity. Hence, the analysis applied to the inlet profiles reveals the explicit impact of swirl on the instability.

The flow at the nozzle lip reveals two instabilities, indicated by two unstable eigenvalues. The first is classified as a shear instability being qualitatively similar for the non-swirling and swirling jet. The other is identified as a centrifugal instability that exists only with swirl. Before entering the presentation of these instabilities, it is important to familiarize the reader with the terms used to differentiate between the different mode alignments.

\subsection{Identifying the direction of winding and rotation of the helical modes}

According to the normal mode decomposition introduced in (4.2), instability modes are equally represented by

$$
(\alpha, m, \omega) \text { or }\left(-\alpha^{*},-m,-\omega^{*}\right) .
$$

Without loss of generality, we consider only mean flows with $V_{\theta} \geqslant 0$. Cases with negative $V_{\theta}$ can be deduced by the following symmetry (Olendraru \& Sellier 2002; Gallaire \& Chomaz 2003b)

$$
\left(V_{\theta}, m\right) \rightarrow\left(-V_{\theta},-m\right)
$$

In experimental studies, it is common to let $\omega_{r}$ and $\alpha_{r}$ only take positive values (e.g. see Panda \& McLaughlin 1994; Liang \& Maxworthy 2005). The phase function $\left(\alpha_{r} x+\right.$ $\left.m \theta-\omega_{r} t\right)$ then implies that at a fixed axial location $x$, modes with $m>0$ rotate in time in the direction of the basic flow and are called co-rotating, while modes with $m<0$ are called counter-rotating. The phase function further implies that at fixed time $t$ and increasing $x$, modes with $m>0$ have a line with constant phase that winds in opposite direction to the basic flow rotation and are called counter-winding and modes with negative $m$ are called co-winding. Although the restriction to positive frequencies and wavenumbers appears more intuitive, it constrains the investigation of certain spatiotemporal mode configurations that may occur in reality. It is therefore more general to let $\alpha_{r}$ take both signs. In that case, co-winding modes correspond to $\alpha_{r} m<0$ and counter-winding modes to $\alpha_{r} m>0$. Hence, a co-rotating co-winding mode corresponds to positive $m$ and $\omega_{r}$ and negative $\alpha_{r}$. These modes exist especially in swirling jets and they can only be expressed by negative wavenumbers. In fact, for the presentation of certain diagrams, it is convenient to also let $\omega_{r}$ take both signs with negative values being derived from (5.1). This implies that co-rotating modes refer to $\omega_{r} m>0$ and counter-rotating modes to $\omega_{r} m<0$. In figure 8 the six non-axisymmetric mode variants are summarized in a schematic drawing. It may serve as a visual aid that comes in handy during the discussion of the stability analysis.

\subsection{Shear instability: dominating the non-swirling and swirling jet}

Figure 9 provides an overview of the influence of swirl on the shear instability. The rather unusual plotting style appears several times throughout this work and is therefore described in more detail at this point. The filled contours represent the dimensionless spatial growth rate $-\alpha_{i} \delta_{x}$ computed for various dimensionless frequencies $\omega_{r} \delta_{x} / V$ and azimuthal wavenumbers $m$. The contour levels are always the same within each figure. Regions of negative growth rate $\left(-\alpha_{i}<0\right)$ are blanked. 


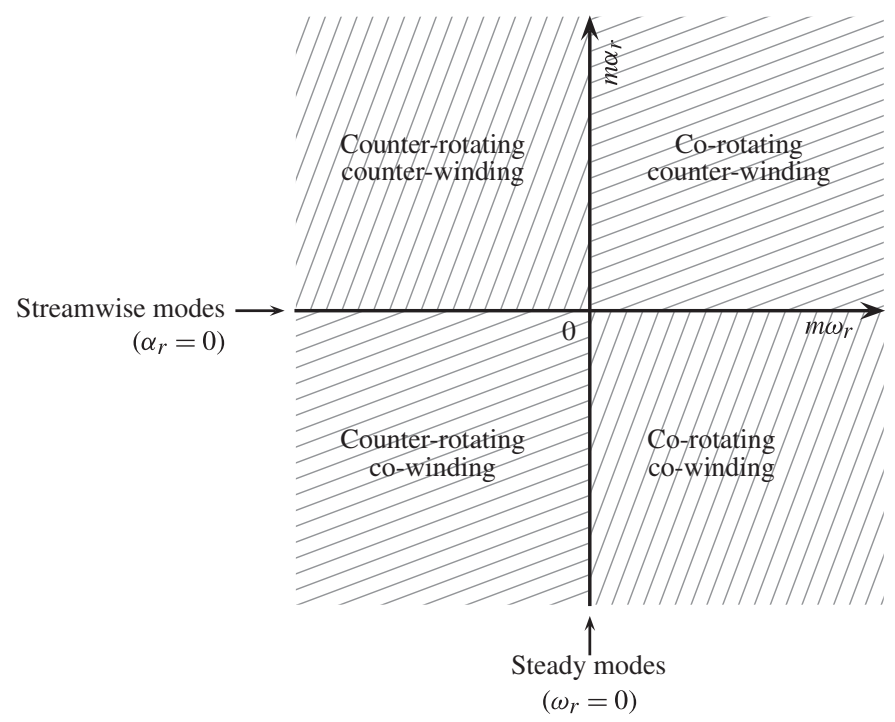

FIGURE 8. Schematic drawing of the six non-axisymmetric variants of mode alignments in swirling jets; $m, \omega_{r}$, and $\alpha_{r}$ may take both signs and are equally expressed by $\left(\alpha_{r}, m, \omega_{r}\right)$ or $\left(-\alpha_{r},-m,-\omega_{r}\right)$.

The labelled contour lines refer to the dimensionless axial wavenumbers $\alpha_{r} \delta_{x}$ and are also only displayed for unstable modes. For better visibility, the contour lines of $\alpha_{r} \delta_{x}$ are black, while the lines separating the filled contours of $-\alpha_{i} \delta_{x}$ are light grey. For most cases, we allow the frequency and axial wavenumber to have positive and negative values. The symmetry of the $-\alpha_{i} \delta_{x}$ contours with respect to the origin of the $\omega_{r}-m$-plane indicates redundancies for this plotting style (see transformation (5.1)).

Referring to figure $9(a)$, the non-swirling jet is most unstable to axisymmetric disturbances in agreement with previous investigations (see e.g. Crighton \& Gaster 1976; Cohen \& Wygnanski 1987a; Gallaire \& Chomaz 2003b). Co-rotating counterwinding modes $\left(\omega_{r} m>0, \alpha_{r} m>0\right)$ and counter-rotating co-winding modes $\left(\omega_{r} m<0\right.$, $\left.m \alpha_{r}<0\right)$ are equally unstable. The streamwise wavenumber of maximum amplification is approximately $\alpha_{r}^{\max } \delta_{x}=0.2$ and remains constant for varying $m$. The overall maximum amplification and corresponding frequency agrees well with the values reported by Cohen \& Wygnanski (1987a). This brings credibility to the measured mean flow on which the analysis is based.

By imposing swirl onto the flow, the symmetry breaks (figure 9b-d) and counter-rotating modes $\left(\omega_{r} m<0\right)$ undergo different amplification than co-rotating modes $\left(\omega_{r} m>0\right)$. The maximum growth rate of the axisymmetric modes $(m=0)$ is very similar to the non-swirling jet as this mode is purely driven by the axial shear. Surprisingly, also the maximum growth rates and the associated streamwise wavenumbers for $m \neq 0$ are nearly unaffected by the strength of the swirl component. This indicates that also the most amplified helical modes of this instability are driven by the axial shear layer similar to the Kelvin-Helmholtz instability of the non-swirling baseline case. However, there is yet a considerable influence of the swirl component on the most amplified frequencies. The contours of the growth rates and the streamwise wavenumbers depicted in figure 9 become successively tilted with increasing swirl, resulting in higher frequencies for the co-rotating modes 


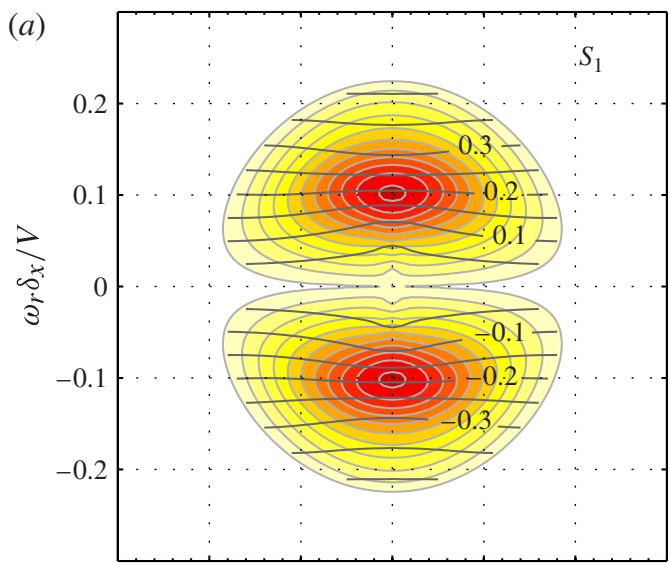

(b)

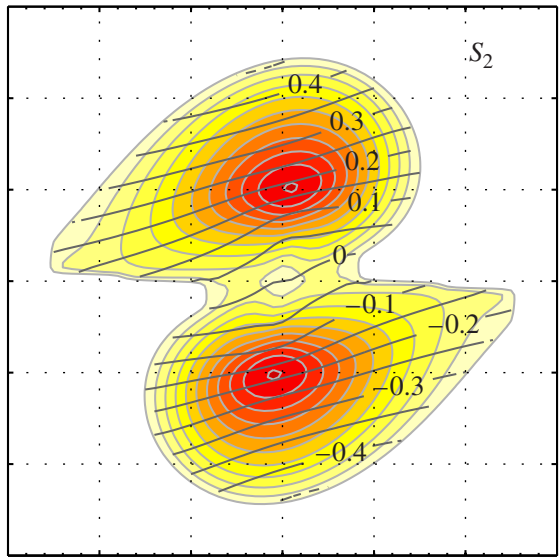

(c)

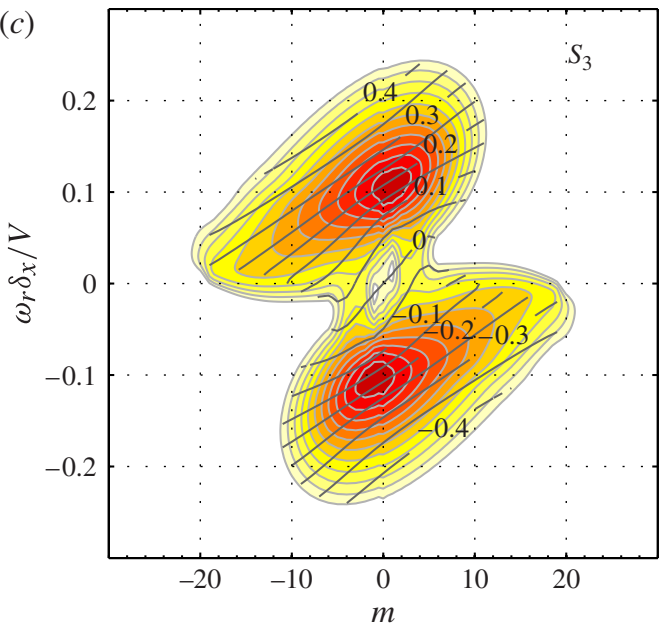

(d)

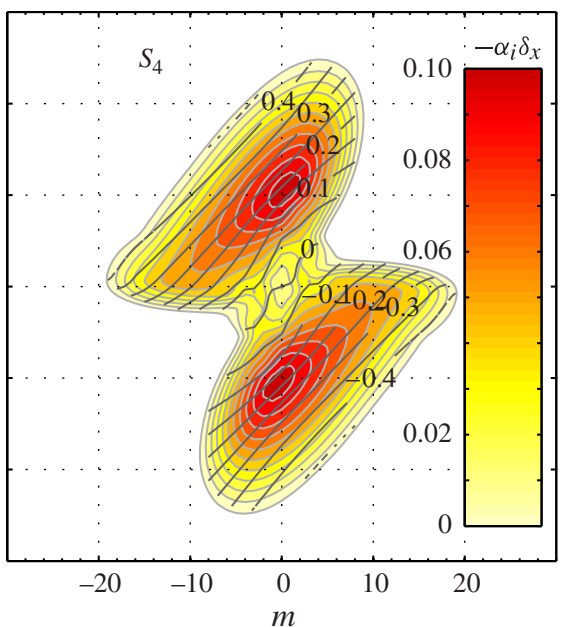

FIGURE 9. (Colour online) Instability of the shear mode at the nozzle exit $(x / D=0)$ for the non-swirling jet $(a)$ and the swirling jets $(b-d)$. Filled contours refer to the spatial growth rate $-\alpha_{i} \delta_{x}$. Dark-grey labelled contour lines refer to the streamwise wavelength $-\alpha_{r} \delta_{x}$. Stable modes $-\alpha_{i} \delta_{x}<0$ are blanked. Quantities are derived numerically for varying azimuthal wavenumbers $m$ and frequencies $\omega_{r}$.

and lower frequencies for counter-rotating modes. This leads to a destabilization of steady co-winding modes $\left(\omega_{r}=0, \alpha_{r} m<0\right)$, co-rotating co-winding modes $\left(\omega_{r} m>0\right.$, $\left.\alpha_{r} m<0\right)$ and co-rotating streamwise modes $\left(\omega_{r} m>0, \alpha_{r}=0\right)$, which are all stable without swirl.

The swirl-induced tilt of the contours shown in figure 9 is directly related to the rotation of the base flow. In a swirling jet, a disturbance initiated at the nozzle lip is convected in axial and azimuthal direction, which implies that the group velocity of a travelling wave has a non-zero axial and azimuthal component. The group rotation rate defined by $\Omega_{g r}=\left(\partial \omega_{r} / \partial m\right)_{\alpha_{r}=\text { const. }}$ represents the slope of the $\alpha_{r} \delta_{x}$ contour lines shown in figure 9. Consistently, it increases successively with increasing swirl for all unstable modes. The non-zero $\Omega_{g r}$ for swirling jets is supported by the experimental investigation of the impulse response that is discussed in $\S 8$. 


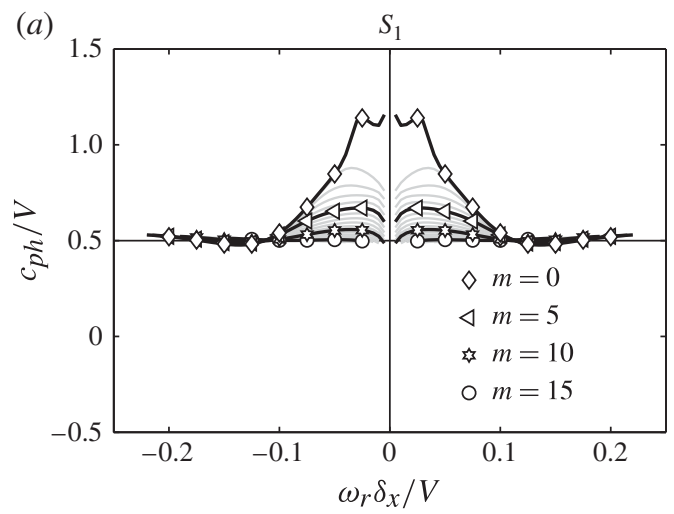

(b)

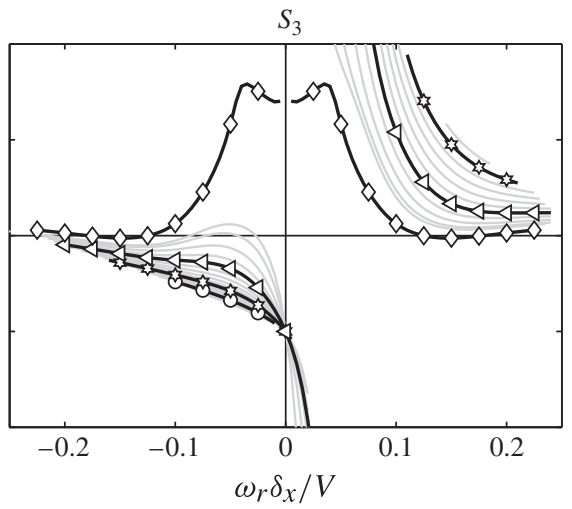

FIGURE 10. The streamwise phase velocity $c_{p h}=\omega_{r} / \alpha_{r}$ versus the frequency $\omega_{r}$ of the shear mode; only unstable modes are shown.

The non-zero group rotation rate induced by the swirl significantly affects the streamwise phase velocity of the instabilities. Figure 10 shows $c_{p h}=\omega_{r} / \alpha_{r}$ for the non-swirling $\left(S_{1}\right)$ and the swirling $\left(S_{3}\right)$ jet with the first revealing typical features of the Kelvin-Helmholtz instability. For frequencies near neutral amplification, the instability waves are only weakly dispersive with $c_{p h} / V \approx 0.55$. The non-dispersiveness enables nonlinear modal interactions to occur, such as modes resonating with their subharmonics (Cohen \& Wygnanski 1987a; Paschereit, Wygnanski \& Fiedler 1995) or triad resonance between two spinning modes (Long \& Petersen 1992). These mechanisms do not apply as rigourously to the swirling jet. Figure 10 reveals a distinct dependence of the phase velocity on $\omega_{r} \delta_{x} / V$ and $m$, which hampers the intermodal resonance. With swirl, the resonance condition of equal phase velocity (between the same or different $m$ ) is only fulfilled for a short streamwise extent that might be insufficient for an effective resonance to build up. However, it should be recalled here that in a non-parallel flow, the phase velocity is also a function of $r$ and the resonance condition can still be fulfilled for a certain $r$ only.

Finally, the radial velocity magnitude distribution of the shear modes, given by their eigenfunctions, are shown in figure 11 for the non-swirling $\left(S_{1}\right)$ and swirling jet $\left(S_{3}\right)$. The radial coordinate is centred to the jet half-width $R_{.5}$ and normalized with respect to the momentum thickness $\delta_{x}$. Only the modes at maximum growth rate for a given $m$ and positive frequency are shown. The eigenfunctions are not significantly altered by the swirl. The velocity magnitude peaks approximately at the centre of the axial shear layer $\left(r=R_{.5}\right)$, indicating the importance of the axial shear layer for this type of instability.

Concluding this section, the most unstable modes at the nozzle exit are driven by a shear instability, with the overall maximum spatial growth rate determined by the shape of the axial velocity profile. The selection of the most amplified axial wavenumbers $\alpha_{r}^{\max }$ depends only weakly on $S$ and $m$. However, the corresponding frequencies $\omega_{r}^{\max }$ depend strongly on $S$ and $m$ due to the non-zero azimuthal group rotation rate $\Omega_{g r}$ caused by the swirling motion of the mean flow. This dependence is a kinematic effect, as stated by Martin \& Meiburg (1994), and can be illustrated by considering inclined waves on a infinite long cylinder as a model for instability waves travelling along a swirling jet. Consider first waves fixed to the rotating cylinder. For a given wavenumber (here $\alpha_{r}^{\max }$ ), the axial phase velocity depends on 

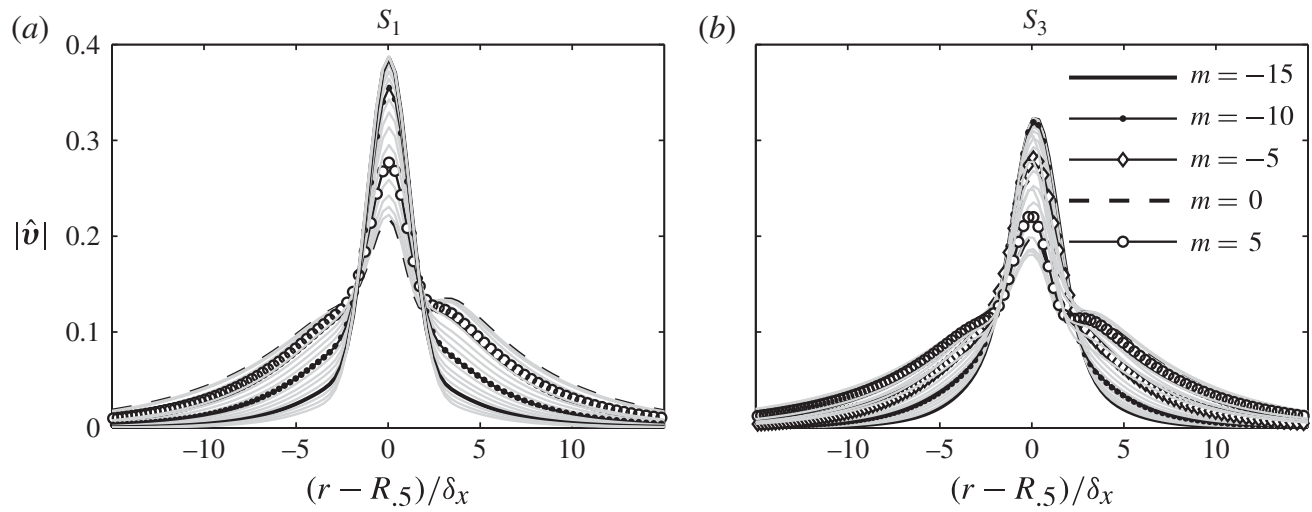

FIGURE 11. Amplitude distribution of the shear modes for the non-swirling jet $S_{1}$ and the swirling jet $S_{3}$ calculated for positive frequencies $\omega_{r}^{\max }$ at maximum spatial amplification.

the rotation rate of the cylinder (here $\Omega_{g r}$ or $S$ ) and the inclination of the waves $\left(m / \alpha_{r}^{\max }\right)$, yielding $c_{p h} \propto \Omega_{g r} m / \alpha_{r}^{\max }$. Now, consider a non-rotating cylinder that moves in axial direction at constant velocity (here $V / 2$ ). The phase velocity is then given by $c_{p h}=V / 2$, independent of the inclination. Superimposing these two cases results in the relation $c_{p h} \propto V / 2+\Omega_{g r} m / \alpha_{r}^{\max }$ and with $c_{p h}=\omega_{r} / \alpha_{r}^{\max }$ we get the proportionality $\omega_{r} \propto V / 2 \alpha_{r}^{\max }+\Omega_{g r} m$, which describes the influence of swirl on $\omega_{r}$ and $m$ of the most unstable wavenumber $\alpha_{r}^{\max }$.

\subsection{Centrifugal instability}

In addition to the shear instability discussed above, a second disturbance mode is observed that only exist in the presence of swirl. Figure 12 shows the corresponding contours of $-\alpha_{i} \delta_{x}$ in the $\omega_{r}-m$ plane for different swirl intensities. Its overall maximum growth rate increases successively with increasing swirl, however, reaching no larger values than a third of the maximum growth rate of the shear mode. Counter-rotating co-winding modes $\left(\omega_{r} m<0, \alpha_{r} m<0\right)$ are promoted by the instability with $m=-7$ and $\omega_{r} \delta_{x} / V \approx 0.3$ being most unstable for $S_{2}$ and $m=-2$ and $\omega_{r} \delta_{x} / V \approx 0.5$ for $S_{3}$ and $S_{4}$. The streamwise wavenumber $\alpha_{r}$, indicated as black contour lines in figure 12, reveals much smaller wavelengths in regions of maximum amplification in comparison with the shear instability. This type of instability selects short axial and azimuthal wavelengths at high frequencies promoting flow structures at smaller scales than the shear mode. Moreover, this type of instability does not promote steady or streamwise modes that are found in the shear mode.

The tendency of this second, less unstable perturbation to counter-rotating modes at small wavelengths indicates a manifestation of a centrifugal instability. Rayleigh's well-known inviscid criterion provides a necessary and sufficient condition for a swirl profile to be centrifugally unstable to axisymmetric perturbations (Drazin \& Reid 2004). The Rayleigh criterion was generalized to vortices with axial flow by Howard \& Gupta (1962) and extended to three-dimensional perturbations by Leibovich \& Stewartson (1983). According to their inviscid asymptotic study, the swirling jet becomes centrifugally unstable if

$$
\sigma^{2}(r)=\frac{2 V_{\theta}\left(r V_{\theta}^{\prime}-V_{\theta}\right)\left(V_{\theta}^{2} / r^{2}-\left(V_{\theta}^{\prime}\right)^{2}-\left(V_{x}^{\prime}\right)^{2}\right)}{\left(r V_{\theta}^{\prime}-V_{\theta}\right)^{2}+\left(r V_{x}^{\prime}\right)^{2}}>0,
$$




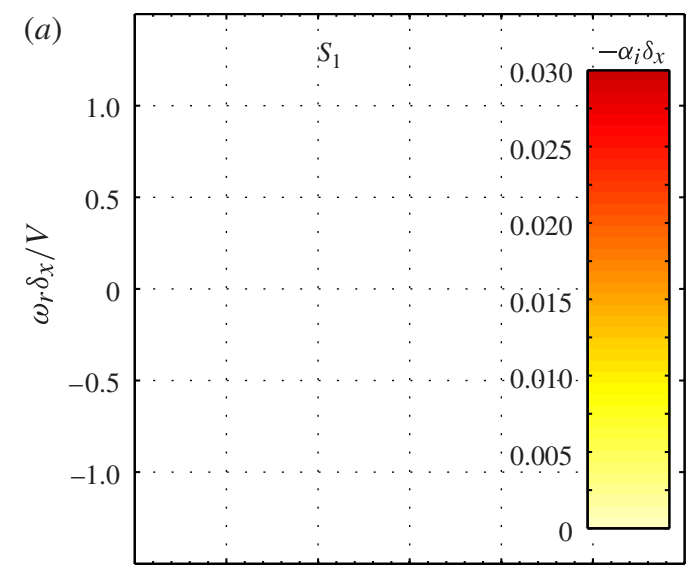

(b)
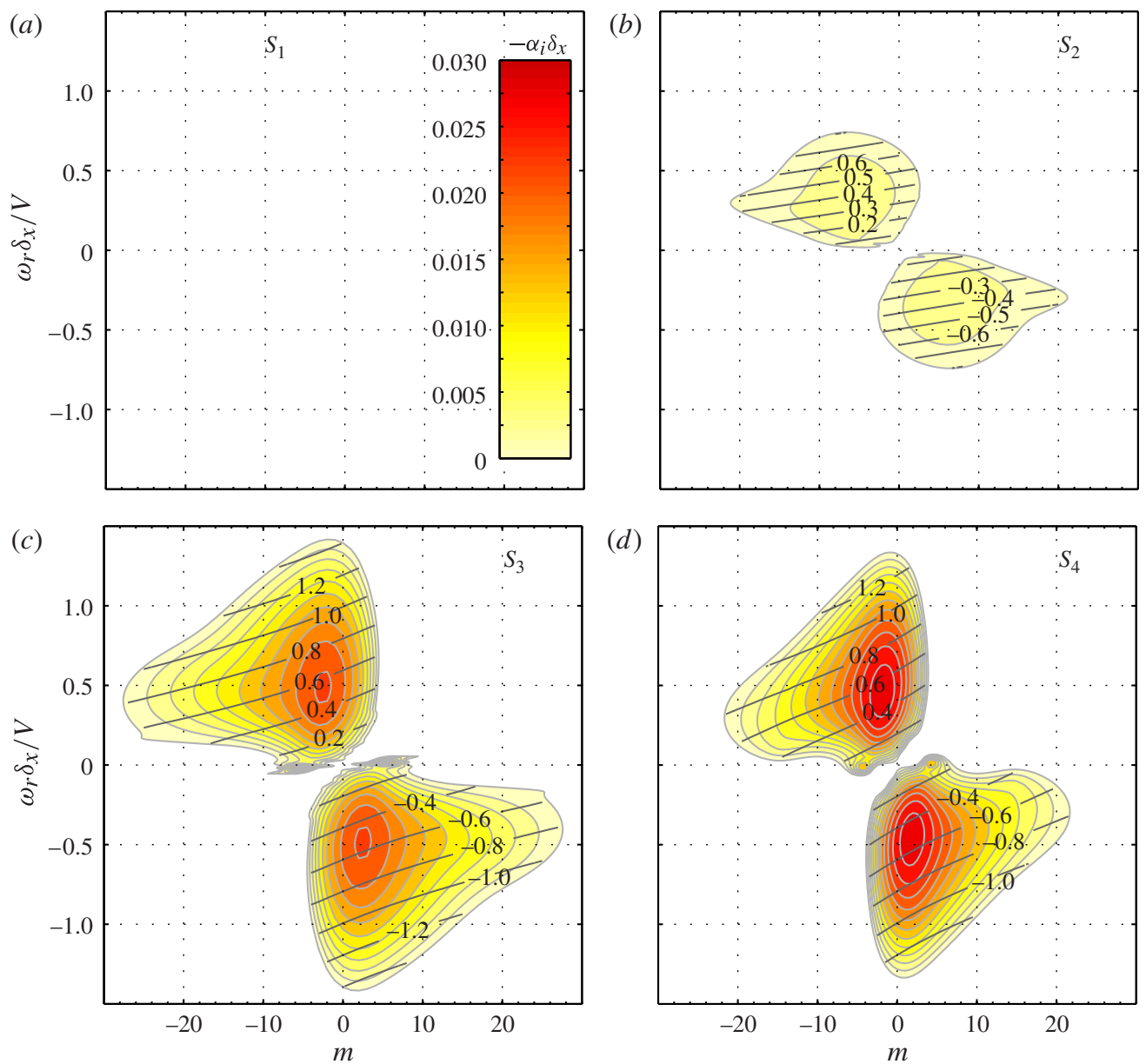

FIgURE 12. (Colour online) Instability of centrifugal mode at $x / D=0$ for the non-swirling jet $\left(S_{1}\right)$ and the swirling jets $\left(S_{2}-S_{4}\right)$. The plots are the same style as in figure 9.

where the prime indicates the first spatial derivative in the radial direction. If the equality is satisfied, the criterion provides the square of the temporal growth rate $\sigma(r)$ of the most unstable mode centred at the radius $r$. Figure 13 shows this quantity for the flow at the nozzle lip. The maximum temporal growth rate $\sigma_{\max }$ follows the same trend as the maximum spatial growth rate shown in figure 12.

The radial position of $\sigma_{\max }$ corresponds to the characteristic radius $R_{c}$ of the most (temporally) unstable mode. As shown in figure 14, this radius marks quite well the peak amplitude of the most (spatially) amplified counter-rotating modes derived from the stability analysis. The centrifugal modes peak at radial positions that do not coincide with the centre of the axial shear layer $R_{.5}$ (black asterisk in figure 14), where the shear modes peak. Moreover, the centrifugal modes shown in figure 14 are not all located at the same radius. This is in accordance with Leibovich \& Stewartson (1983), stating that the eigenmodes are centred at the radial position where the helix angle $\beta=-\alpha_{r} / m$ is aligned with the direction of the zero rate of strain, which varies for each wavenumber pair. 


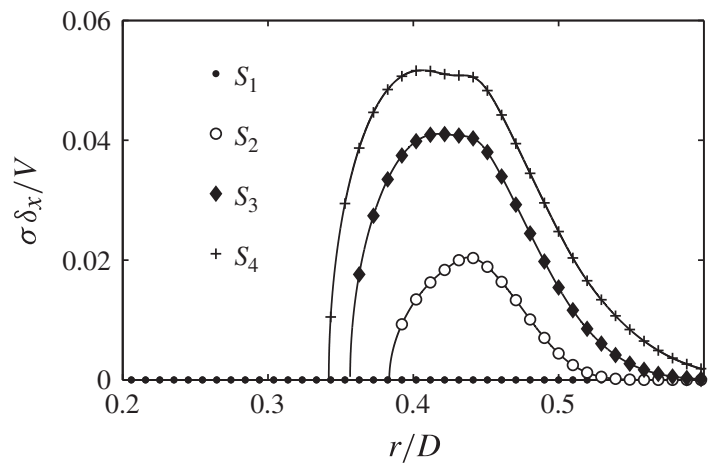

FIGURE 13. Radial distribution of the temporal growth rate $\sigma$ at the nozzle lip predicted from the Leibovich-Stewartson instability criterion.

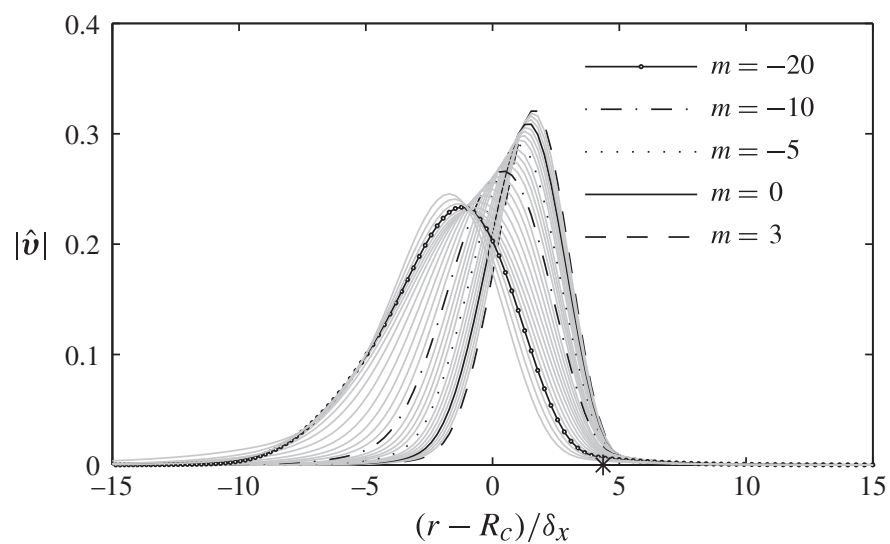

FIGURE 14. Amplitude distribution of the centrifugal mode at the nozzle exit $(x / D=$ 0 ) for the swirling jet $S_{3}$ calculated for positive frequencies $\omega_{r}^{\max }$ at maximum spatial amplification. Radial coordinate is off-set by the characteristic radius $R_{c}$ derived from the Leibovich-Stewartson stability criterion. The black star marks the radial coordinate of the centre of the axial shear layer $R_{.5}$.

To conclude, the second, less unstable perturbation that exists only for the swirling jet follows the trend predicted by the inviscid stability criterion of Leibovich \& Stewartson (1983), suggesting that it is a manifestation of a centrifugal type instability. Its characteristic (wavelength, frequency, amplitude distribution) differs significantly from the shear mode and is, therefore, clearly distinguishable. Moreover, it shows strong qualitative similarities with instabilities classified as centrifugal type in previous investigations (Lu \& Lele 1999; Cooper \& Peake 2002; Müller \& Kleiser 2008). The impact of viscosity seems to be negligible at the nozzle where the eddy viscosity is relatively small.

\section{Streamwise evolution of instability}

In this section, the downstream development of the shear instability and the centrifugal type instability are investigated. Owing to the divergence of the jet, the mean flow and corresponding stability characteristics change significantly in 
streamwise direction, and certain modes stabilize with downstream distance and die away while others remain unstable. To obtain the overall growth of the instability modes, it is necessary to perform the stability analysis at various streamwise locations.

\subsection{Shear instability}

The Kelvin-Helmholtz instability in axisymmetric non-swirling jets has been investigated by numerous researchers, and it will serve as a benchmark to validate the present numerical results. At the nozzle exit, where the shear layer is thin, the non-swirling jet is known to be unstable to various axial and azimuthal wavenumbers and frequencies (see e.g. Cohen \& Wygnanski 1987a). With increasing distance from the nozzle, the number of unstable azimuthal modes decreases successively, and at the end of the potential core, only the bending modes with $m= \pm 1$ remain unstable. The present investigation confirms these results.

Figure 15 displays the contours of spatial growth rate in the $\omega_{r}-x$ plane for $0 \leqslant m \leqslant 3$. Recall that in the absence of swirl, co-rotating counter-winding modes are equally unstable than counter-rotating co-winding modes and, hence, we can restrict their presentation to positive $m$ and $\omega_{r}$. The axisymmetric mode, which is most unstable at the nozzle exit, stabilizes at $x / D \approx 3$ (figure $15 a$ ). All non-axisymmetric modes except for $m= \pm 1$ also stabilize in downstream direction with their neutral point located closer to the nozzle for higher $m$. The frequency and wavelength of the most unstable mode remains approximately constant with $x$, indicating the appropriate choice of the velocity and length scale.

The maximum growth rates $\alpha_{i}^{\max }$ are shown explicitly in figure 16 . It can be directly compared with the results from the inviscid analysis conducted by Cohen \& Wygnanski (1987a). Close to the nozzle exit, the agreement is good. However, the viscous analysis presented here predicts a faster downstream decay of $-\alpha_{i}^{\max }$. In the present analysis, the axisymmetric modes $(m=0)$ become neutrally stable at $x / D \approx 3.3$, while the inviscid analysis of Cohen \& Wygnanski (1987a) predicts $x / D \approx 4.5$. Perhaps more importantly, the maximum growth rate of the bending modes with $m= \pm 1$ presented here asymptotes to 0.22 , which is less than half of the value derived by Cohen \& Wygnanski (1987a). The discrepancy between the viscous and the inviscid analysis is due to the eddy viscosity model used in the present investigation. Near the nozzle exit, the turbulent viscosity $v_{t}$ is very small and it has only a marginal effect on the growth of instability. With larger distance from the nozzle, the turbulent fluctuations increase, resulting in a rapid increase of $v_{t}$ and a drastic decay of the growth rate of the instability. The inviscid analysis overestimates the growth rate that is observed in experiments, as shown by Cohen \& Wygnanski $(1987 a)$, while the present approach yields a theoretical prediction that agrees well with the experimental results as shown later.

By introducing swirl to the flow, the symmetry breaks and co-rotating modes undergo different amplification cycles than counter-rotating modes and, hence, contours of $-\alpha_{i} \delta_{x}$ must be derived separately for positive and negative frequencies. The streamwise distribution of $\alpha_{i}$ of the swirling jet configurations $S_{2}$ and $S_{3}$ is shown in figure 17 for $0 \leqslant m \leqslant 3$. During the ensuing discussion, it is more meaningful to classify the instability modes by their sense of winding than by their direction of rotation. In this spirit, the contour line $\alpha_{r}=0$ in figure 17 that refers to streamwise modes is of particular interest as it separates the regime of co-winding modes from the regime of counter-winding modes. It is located in a 'valley' between regimes of high amplification and, thus, the streamwise modes are always less unstable than the 

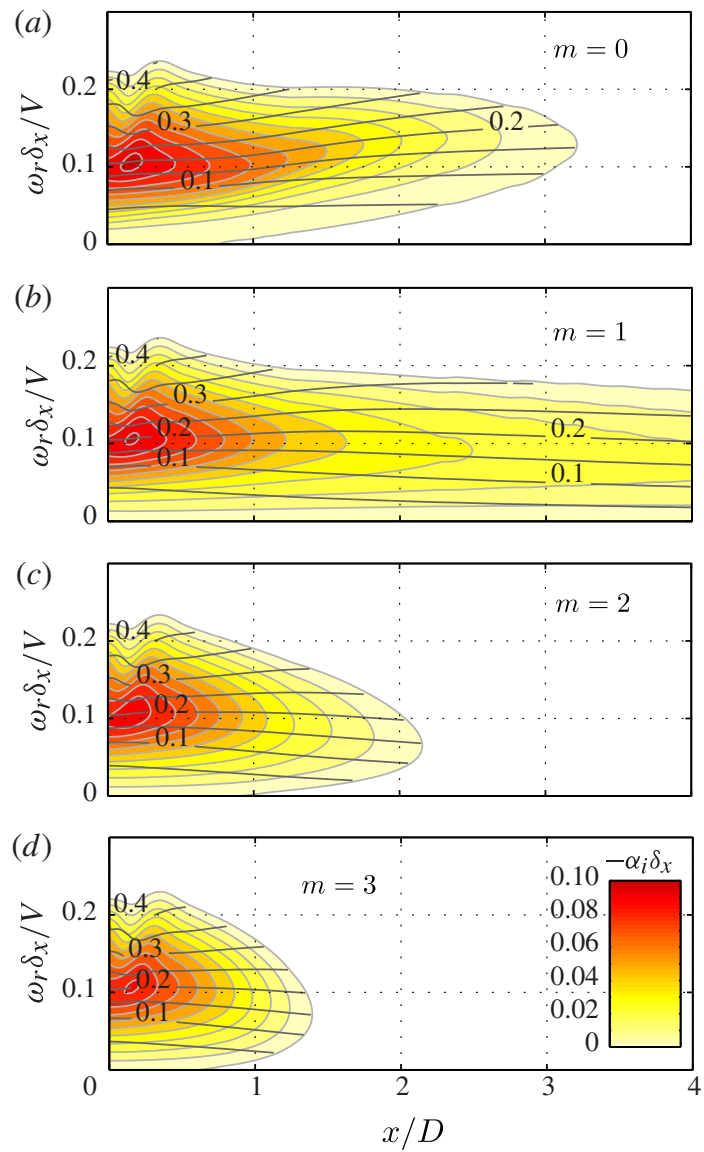

FIGURE 15. (Colour online) Streamwise evolution of spatial growth rate of the shear mode for the non-swirling jet $S_{1}$ for the azimuthal wavenumbers $m=(0,1,2,3)$ (from $a-d)$. Plots are the same style as in figure 9.

most unstable winding modes. Their rotation rate is directly related to the base flow rotation and, thus, their frequency is always larger zero and increases with increasing swirl.

The streamwise evolving flow selects different modes than one would expect from the analysis at the nozzle exit. The counter-winding modes that are most amplified at the nozzle exit do not undergo the strongest overall amplification. At increasing swirl, the growth rates of the counter-winding and axisymmetric modes, which are prominent at the nozzle, reduce rapidly with downstream distance, while the co-winding modes remain unstable for a longer streamwise extent. The magnitude of the streamwise wavenumber of the most amplified modes are approximately constant in the axial direction with $\left|\alpha_{r} \delta_{x}\right| \approx 0.2$, independently of the azimuthal wavenumber (figure 17). This agrees with the non-swirling jet (figure 15). However, the most amplified frequencies are not constant in axial direction unlike the non-swirling jet. For the modes with $m>1$ (figure $17 c-d$ ), the frequency at maximum amplification increases in downstream direction. This results in a destabilization of co-winding steady modes $\left(\omega_{r}=0, \alpha_{r}<0\right)$ at streamwise wavenumbers that are similar to the 


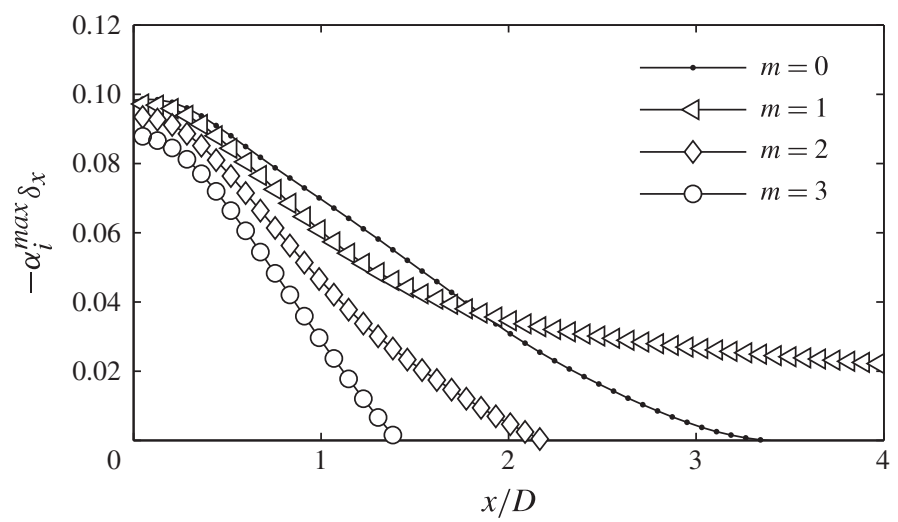

FIGURE 16. Streamwise development of the maximum growth rate $-\alpha_{i}^{\max } \delta_{x}$ of the shear instability for the non-swirling jet $S_{1}$ for different azimuthal mode numbers and positive frequencies.

most amplified ones in the non-swirling jet. At sufficiently strong swirl (right column in figure 17), the $\alpha_{r}=-0.2$ contour line, depicting roughly the most amplified modes, asymptotes the $\omega_{r}=0$ line. This implies that, with increasing downstream distance from the nozzle, the rotation rate of the most amplified co-winding counter-rotating modes decrease continuously until they stand still $\left(\omega_{r}=0\right)$.

The ability to promote steady modes at considerably high amplification rates is a unique feature of swirling jets. The tendency of the co-winding modes to lower rotation rates (frequencies) appears plausible when considering the proportionality $\omega_{r} \propto V / 2 \alpha_{r}^{\max }+\Omega_{g r} m$, derived in $\S 5.2$. It implies that with increasing swirl (increasing $\left.\Omega_{g r}\right)$, the frequency $\omega_{r}$ of the most unstable counter-rotating co-winding mode $(m>0$, $\left.\omega_{r}<0, \alpha_{r}^{\max }<0\right)$ must increase in order to maintain constant axial wavenumber. In the same train of thought, steady modes become most unstable when $V / 2 \alpha_{r}^{\max }=-\Omega_{g_{r}} m$, which seems to be fulfilled at certain axial locations for $m>1$.

Figure 17 reveals that all modes become successively more stable with increasing downstream distance from the nozzle, except for the co-winding modes with $m=1$. Additional computations based on a domain size of $0<x / D<8$ conducted by the authors did not indicate a stabilization of this mode in the farfield. It appears that the swirl-induced destabilization of the co-winding modes and the stabilization of counter-winding modes is maintained for a wide streamwise distance, although the swirl component decays very rapidly in downstream direction (see figure 6). Interestingly, spatial growth of the bending modes asymptote to the same value for all swirl strengths considered, which is twice as high as for the non-swirling jet. The collapse of the growth rates downstream of the potential core is presumably linked to the formation of a universal swirl velocity profile, as indicated by the collapse of the Rossby number, $N_{\theta}$, and $V_{\theta \text {,max }}$ (see figure 6).

Summing up, the swirling jet selects a counter-rotating co-winding $m=1$ mode to be the remaining unstable mode at sufficient downstream distance. This mode is expected to dominate the farfield dynamics. The nearfield is dominated by various co-winding modes with the azimuthal wavenumber $m=2$ to be most amplified. The frequencies of the most amplified modes decay in downstream direction due to the rotational motion of the mean flow, which results in a significant destabilization of steady modes. 
(a)

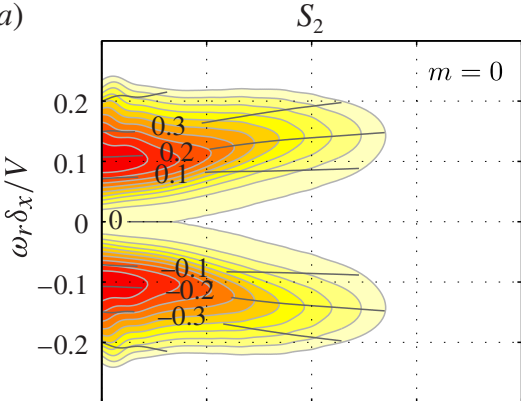

(b)

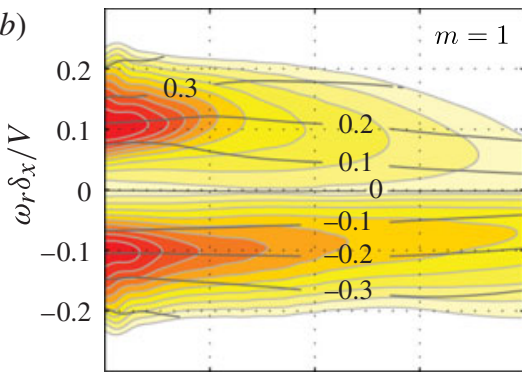

(c)

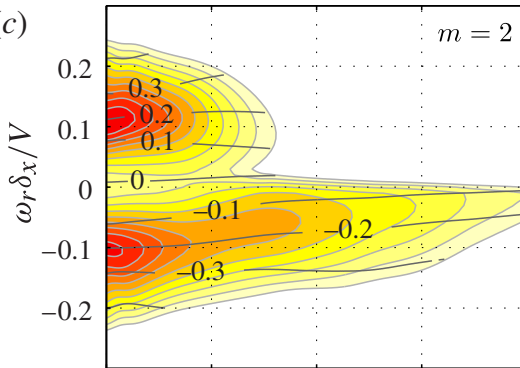

(d)

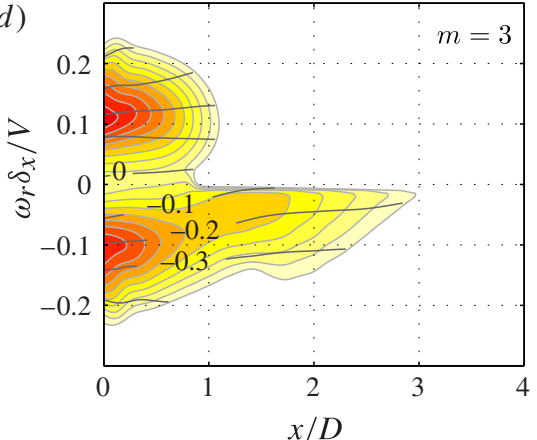

(e)

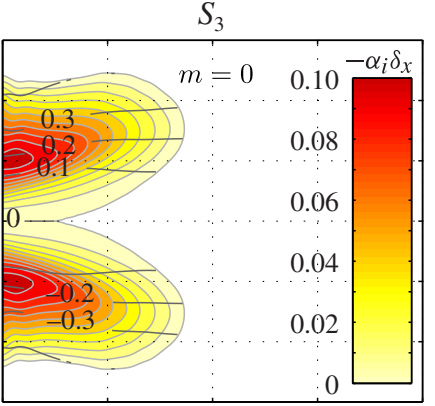

$(f)$

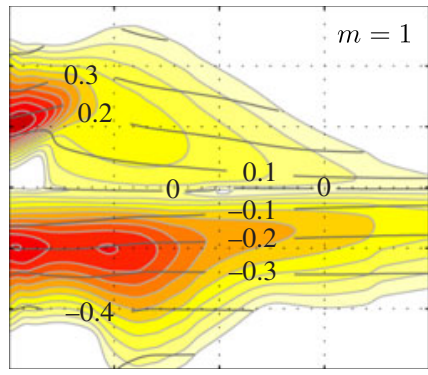

(g)

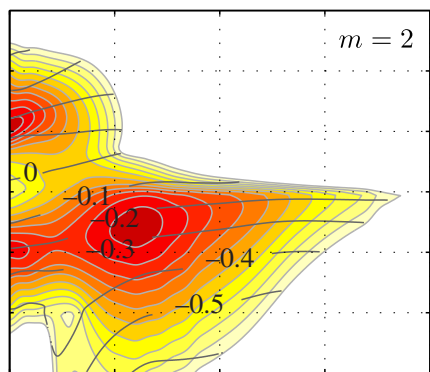

(h)

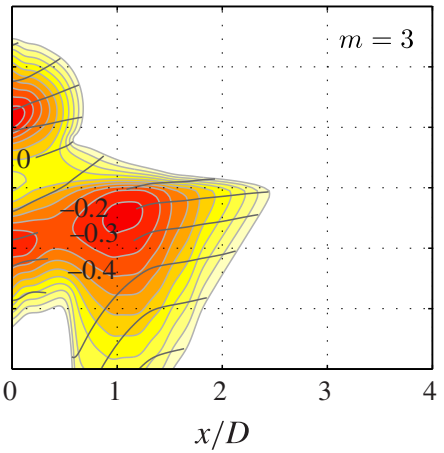

FIgURE 17. (Colour online) Streamwise evolution of spatial growth rate $-\alpha_{i} \delta_{x}$ of the shear mode at azimuthal wavenumbers $m=(0,1,2,3)$ for the swirling jets $S_{2}(a-d)$ and $S_{3}(e-h)$. Plots are the same style as in figure 9 .

\subsection{Centrifugal instability}

At the nozzle the centrifugal instability reveals a tendency to counter-rotating modes at high azimuthal wavenumbers (figure 12). Therefore, the analysis is conducted at various streamwise locations for positive $\omega_{r}$ and for the azimuthal mode numbers 

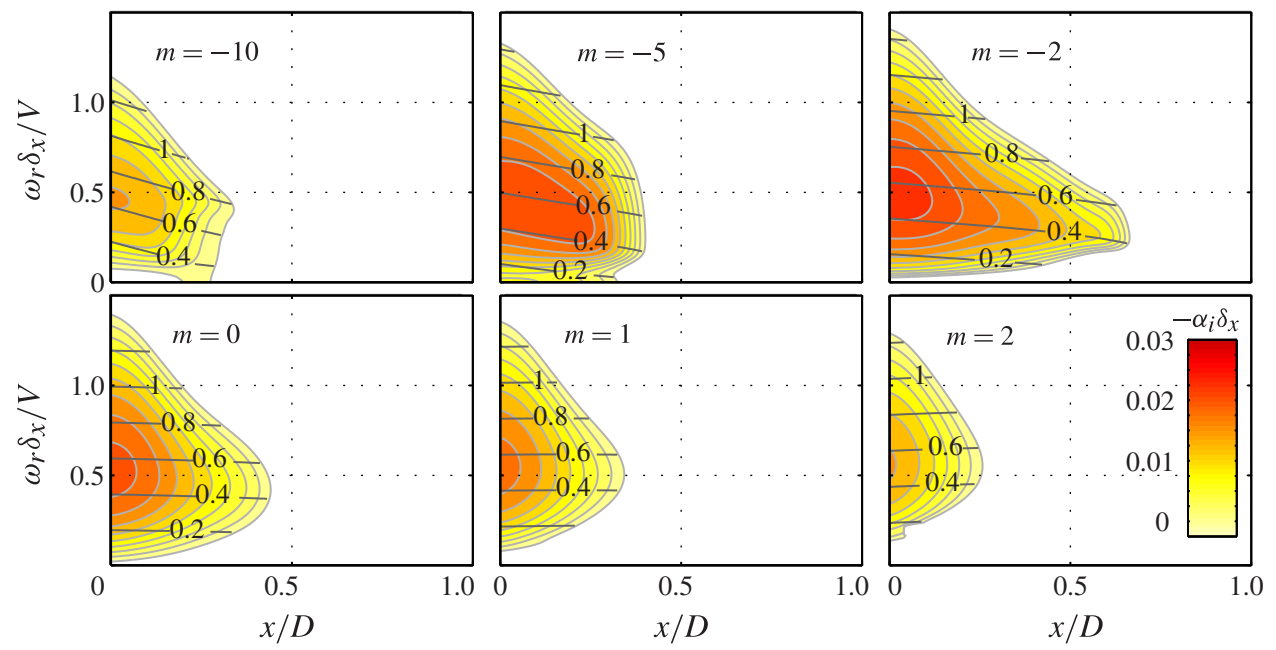

FIgURE 18. (Colour online) Streamwise evolution of spatial growth rate $-\alpha_{i} \delta_{x}$ of the centrifugal mode at azimuthal wavenumbers $m=(-10,-5,-2,-1,0,1,2)$ for the swirling jet $S_{3}$. Same plot style as in figure 9 .

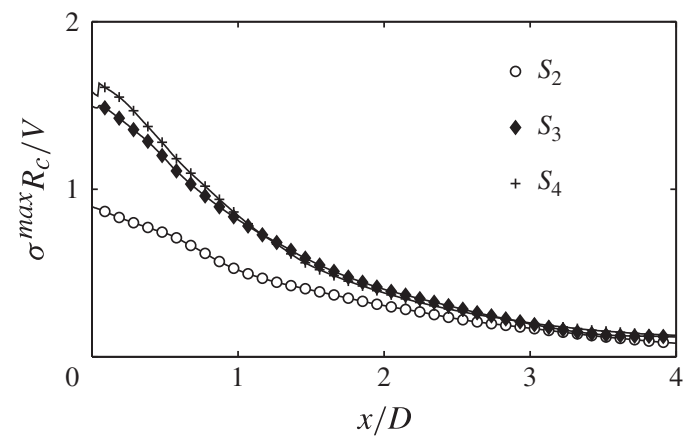

FIGURE 19. Streamwise development of the maximum of the temporal growth rate $\sigma$ predicted from the Leibovich-Stewartson instability criterion.

$m=(-10,-5,-2,0,1,2)$. The results are shown in figure 18. Strikingly, the modes driven by the centrifugal instability decay with downstream direction at a much faster rate than the shear mode, and they all become neutrally stable upstream of $x / D=1$. The stabilization of the centrifugal instability is not in line with the inviscid stability criterion of Leibovich \& Stewartson (1983). Figure 19 shows the streamwise development of the maximum of the temporal growth rate $\sigma$ derived from the inviscid criterion (5.3). Despite its considerable decay shortly downstream of the nozzle, it remains positive throughout. The discrepancy to the linear stability analysis is presumably caused by viscous damping that becomes more significant with downstream distance where the eddy viscosity is higher (see figure 7).

\section{Measurements in the single-mode actuated shear layer}

In a first experiment, the jet at different swirl intensities was forced at a co-rotating mode with $m=1$. The disturbance field of the excited wave was measured with 



FIGURE 20. Radial amplitude distribution of the axial velocity component of the $m=1$ mode for the swirling jet configuration $S_{3}$; markers correspond to hot-wire measurements and lines represent numerical prediction.

hot-wires and compared with the theoretical predictions discussed in the previous sections. Good agreement will bring credibility to the stipulations that are made for the presented stability analysis. Theses assumptions are summarized here again.

(i) The unstable eigenmodes of the mean flow represent the coherent structures.

(ii) Damping due to small-scale turbulence is well modelled by the eddy viscosity.

(iii) The axial overshoot near the nozzle is negligible.

(iv) The quasi-parallel approximation is sufficiently accurate.

In the second experiment, the swirling jet configuration $S_{3}$ was forced at different azimuthal wavenumbers $-2 \leqslant m \leqslant 2$ at a frequency of $100 \mathrm{~Hz}$. This experiment is aimed to validate the dependence of the phase velocity on the azimuthal mode number for the swirling jet.

\subsection{Growth rate of the co-rotating bending mode}

The bending $m=1$ mode was actuated at the frequencies 150,100 and $80 \mathrm{~Hz}$ for the swirl numbers $S_{1}, S_{3}$ and $S_{4}$, respectively. This corresponds to dimensionless frequencies ranging from $\omega_{r} \delta_{x} / V \approx 0.07$ at the nozzle lip to $\omega_{r} \delta_{x} / V \approx 0.4$ at $x / D=1.2$. The frequencies were selected as such that the actuated modes go through their entire amplification cycle within the measurement domain. The frequencies do not correspond to the maximum overall amplification.

The amplitude distribution of the axial component $\left|\hat{v}_{x}\right|$ is derived from the phase-averaged hot-wire measurements and compared with the stability eigenfunctions. Figure 20 shows the amplitude of the co-rotating counter-winding $m=1$ mode at selected axial locations. The black lines refer to the theoretical predictions derived from spatial linear stability analysis, and the symbols refer to phase-locked measurements. The amplitudes are normalized with respect to the area below the graph of the radial extent measured. Good agreement is found upstream of the neutral point of the excited mode, which is at approximately $x / D=0.75$.

The radially integrated amplitude measure $A_{m}(x)$ is derived using (2.3). Figure 21 shows the streamwise amplitude distribution of the modes actuated at $m=1$. The amplitudes are shown in a logarithmic scale and are normalized by their initial value at $x / D=0$. Hence, the graphs represent an amplitude ratio of the radially integrated 


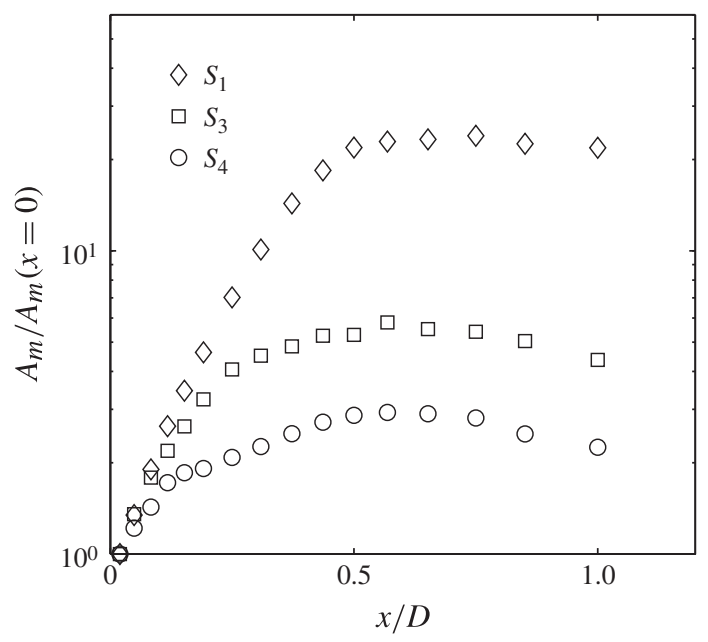

FIGURE 21. Hot-wire measurements of the streamwise amplitude distribution of the actuated co-winding $m=1$ mode for various swirl configurations. Actuation frequencies are 150,100 and $80 \mathrm{~Hz}$ for the swirl numbers $S_{1}, S_{3}$ and $S_{4}$, respectively, to obtain neutral amplification at similar axial locations.

axial velocity component. For all cases, the excited waves saturate at similar axial locations. For the selected forcing frequencies, the waves in the non-swirling jet undergo an overall amplification that is an order of magnitude higher than for the swirling jet.

The spatial growth rate of the axial velocity component is derived from the slope of the amplitude ratio displayed in figure 21. It is displayed in figure 22 for the swirling and non-swirling jet along with the theoretical prediction. The latter is expressed by two different quantities that are both shown in figure 22. The solid black line corresponds to the growth rate of the axial velocity component derived from the reconstructed disturbance field utilizing (4.5), while the dotted line refers to the growth rate of the fictitious parallel flow represented by $-\alpha_{i} \delta_{x}$. Both quantities are equal at the nozzle lip and coincide with the experimental result. Further downstream, the growth rate of the parallel flow overestimates the growth of axial component. This is more pronounced for the swirling jet. The non-uniformity of the flow implies different growth rates for different velocity components, which is not accounted for when considering $-\alpha_{i} \delta_{x}$. The growth rates predicted from the computed disturbance field are reasonably well in line with the experimental data indicating that the negligence of the amplitude scaling $A_{0}$ of the weakly non-parallel correction does not significantly affect the accuracy of the predicted amplitudes.

In conclusion, the local quasi-parallel analysis approximates the streamwise growth of a single-mode excited wave at a sufficient measure of accuracy. The growth rate $-\alpha_{i} \delta_{x}$ of the parallel flow provides an approximate measure of the growth of instability, but for an accurate comparison with experiments the streamwise-varying eigenfunction must be considered. It should be mentioned here that the stability modes presented in this section correspond to the shear instability. The growth rates and eigenfunctions corresponding to the centrifugal instability do not match the experimental results at all (not shown), indicating that they are not excited by the applied actuation. 

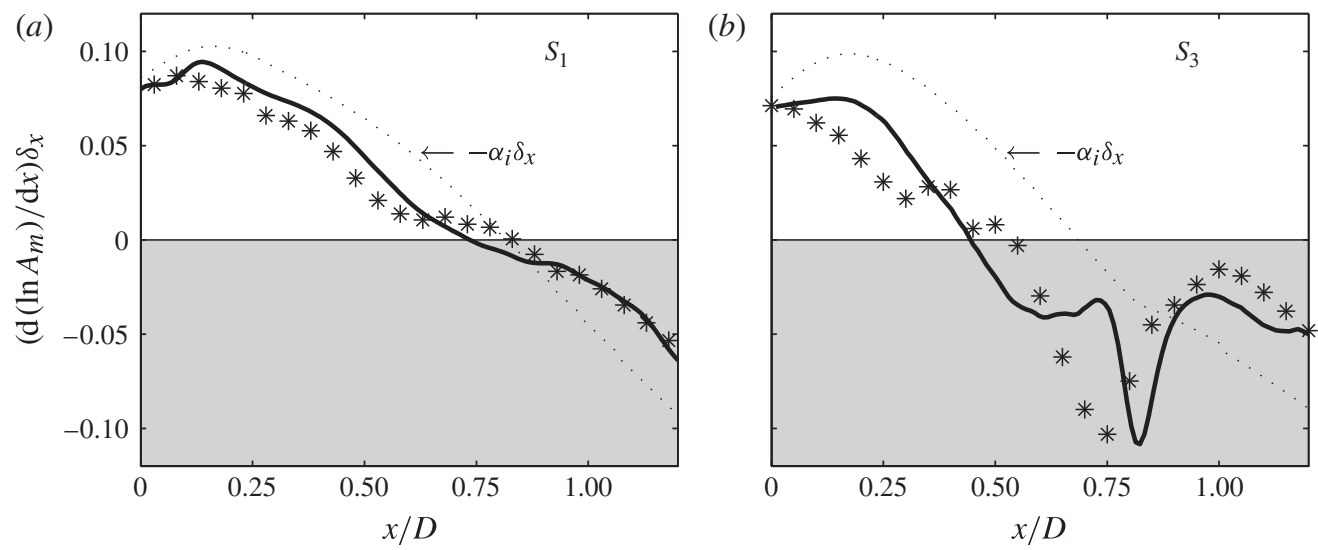

FIGURE 22. Streamwise development of spatial growth rate of actuated co-winding $m=1$ mode for the non-swirling jet $S_{1}(a)$ and the swirling jet $S_{3}(b)$; the black stars refer to hot-wire measurements; the black thick line refers to numerical prediction taking the streamwise variation of the eigenfunction into account and the black dots represent the numerical prediction based on the parallel flow.

\subsection{Streamwise phase velocity for various modes}

The stability analysis predicts a dependence of the axial phase velocity and wavelength on the azimuthal wavenumber when swirl is introduced to the flow. This is validated experimentally by actuating the swirling jet $S_{3}$ at various azimuthal wavenumbers. The corresponding phase distribution $\varphi_{m}(x, r)$ is derived from the phase-locked hot-wire measurements using (2.4). It is depicted in figure 23 for the co-rotating counter-winding travelling waves $m=1$ at several axial locations together with the radial phase distribution obtained from the stability analysis, showing reasonably good agreement.

The phase velocity of the actuated modes is derived from the hot-wire measurements using

$$
c_{p h}(x, r)=\frac{2 \pi f_{a c t}}{\partial \varphi_{m}(x, r) / \partial x}
$$

where $f_{a c t}$ corresponds to the actuation frequency. Note that the phase $\varphi_{m}(x, r)$ is a function of the axial and radial coordinates, and it cannot be condensed to a function of $x$ only, as it is done for the amplitude. However, it is here assumed that the phase velocity in the centre of the shear layer represents the average phase velocity of the entire coherent structure. This approximation is supported by measurements in the forced mixing layer (Gaster et al. 1985). The left graph in figure 24 shows the measured phase delay of modes actuated at $-2 \leqslant m \leqslant 2$ derived at the centre of the axial shear layer $R_{.5}$ for the swirling jet $S_{3}$. All modes are excited at the same frequency. The phase velocity is derived from the slope of the phase delay and displayed in the graph on the right side of figure 24. Accordingly, for lower $m$, the phase increases more rapidly with downstream distance and, consequently, modes at lower $m$ have lower axial phase velocities than modes with higher $m$. This confirms the results from the stability analysis. 


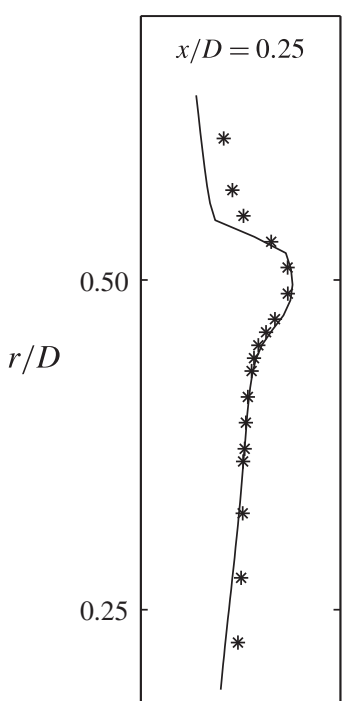

Phase

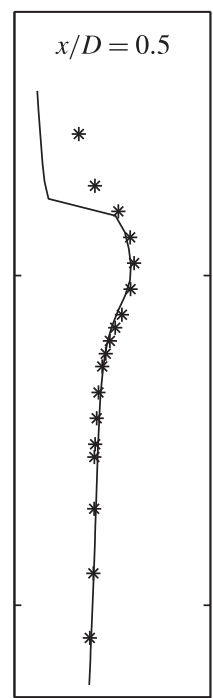

Phase

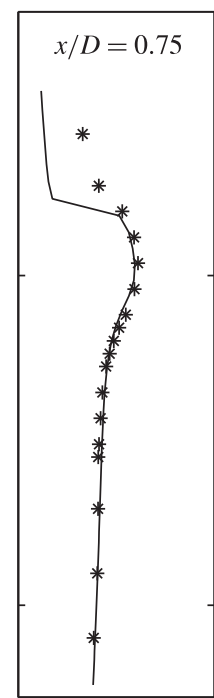

Phase

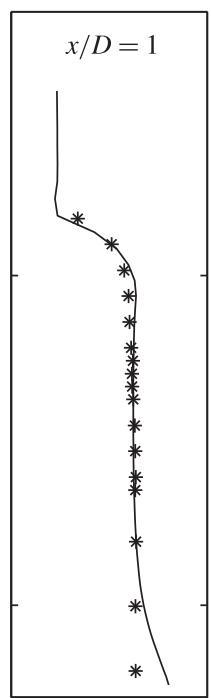

Phase

FIgURE 23. Phase distribution of mode $m=1$ for the swirling jet $S_{3}$, markers correspond to hot-wire measurements, lines represent numerical prediction.
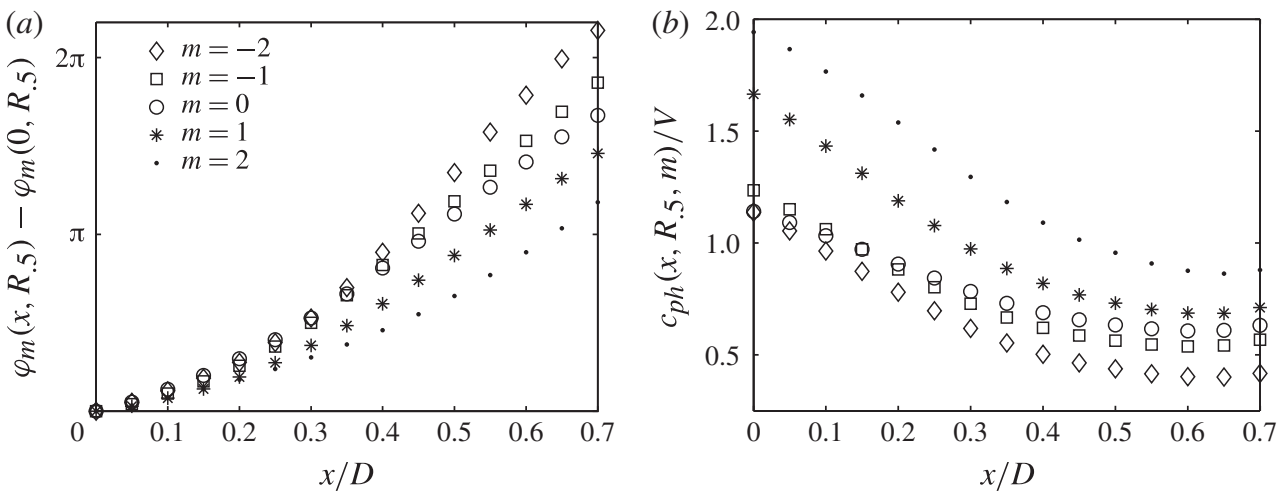

FIgURE 24. Hot-wire measurements of the streamwise evolution of the phase delay $(a)$ and phase velocity $(b)$ for modes excited at various azimuthal wavenumbers $m$ for the swirling jet $S_{3}$. Values are taken in the centre of the axial shear layer $R_{5.5}$.

\section{Measurements in the pulse actuated shear layer}

In the second experiment the response of the shear layer to a single pulse is investigated. The pulse creates a wave packet that consists initially of an infinite number of stable and unstable modes. While propagating downstream, the modes in the wave packet grow or decay according to the stability of the base flow. The modal decomposition of the wave-packet signal reveals the streamwise development of the dominant instabilities, which will serve as a validation of the mode selection predicted from the local stability analysis.

The development of the wave-packet envelope is described first, revealing the shape, the location and the velocity of the wave packet. Thereafter, the modal content of 
the wave packet is discussed and compared with the spatial stability analysis. Results are shown for the non-swirling jet $S_{1}$ and the swirling jet $S_{3}$. It is noted here that in the present experiment, the loudspeaker generates a pulse with an azimuthal extent of $\Delta \theta=\pi / 4$ and an axial extent of $\Delta x / D=0.02$, which differs significantly from a delta function in space. This introduces forcing artifacts that influence the development of the wave packet between the nozzle lip and $x / D=0.25$. Further downstream, the influence on the particular forcing characteristic becomes less significant and the wavepacket evolution is shown to be in line with the theoretical prediction.

\subsection{Trajectory of the wave-packet envelope}

In general, the Fourier coefficients of a signal $v\left(t_{j}\right)$ sampled at $j=1 \ldots N$ time points are given by

$$
\hat{v}(n)=\frac{1}{N / 2} \sum_{j=0}^{N} v\left(t_{j}\right) \exp \left(-\mathrm{i} \frac{\pi n j}{N / 2}\right)
$$

and the envelope of the signal is given by

$$
v^{e}\left(t_{j}\right)=\left|\sum_{n=0}^{N / 2} \hat{v}_{n} \exp \left(\mathrm{i} \frac{\pi n j}{N / 2}\right)\right| .
$$

The measured signal of the wave packet is represented by the coherent axial velocity component $v_{x}^{c}(\boldsymbol{x}, t)$. The experimental arrangement allows for a very good temporal resolution, a reasonably good spatial resolution in radial direction and, due to the specific ensemble-averaging procedure, a good spatial resolution in azimuthal direction. The resolution in axial direction is poor, as data are acquired only for the streamwise locations $x / D=(0.02,0.25,0.6,1,1.5,2,2.5,3)$. Hence, the signal of the wave packet is Fourier decomposed in $r, \theta$ and $t$ direction for each streamwise position, separately, and the corresponding envelope $v_{x}^{e}(\boldsymbol{x}, t)$ is derived from the inverse three-dimensional Fourier transformation, in accordance with (8.2). Consistent with the single-mode investigation, the radial dependence of the envelope is omitted by integrating the envelope across the axial shear layer, yielding the following expression for the envelope amplitude

$$
A^{e}(x, \theta, t)=\frac{1}{D}\left(\int_{R_{.05}}^{R .95}\left(v_{x}^{e}\right)^{2} r \mathrm{~d} r\right)^{1 / 2}
$$

Figure 25 shows the trajectory of the wave packet envelope in a three-dimensional plot for the non-swirling jet $S_{1}$ and the swirling jet $S_{3}$. Two-dimensional contours are displayed for each streamwise measurement location, showing the envelope amplitude distribution along the $\theta-t$ plane. The time is made dimensionless using the half bulk velocity $V / 2$, which is approximately the streamwise convection velocity of the disturbance. At each axial location, $A^{e}$ is normalized by its maximum value $A_{\max }^{e}$ within the corresponding $\theta-t$ plane. This normalization facilitates the comparison of the wave packet envelope at different crosswise measurement planes. Contour surfaces with $A^{e} / A_{\max }^{e}<0.5$ are blanked and the lowest contour line $A^{e} / A_{\max }^{e}=0.5$ is selected arbitrarily as the characteristic outer bound of the wave packet. The corresponding coordinates of the leading and trailing edge of the wave packet are projected on the axis planes (see dotted lines at $x / D=1.5$ ). They are marked by white-filled black 

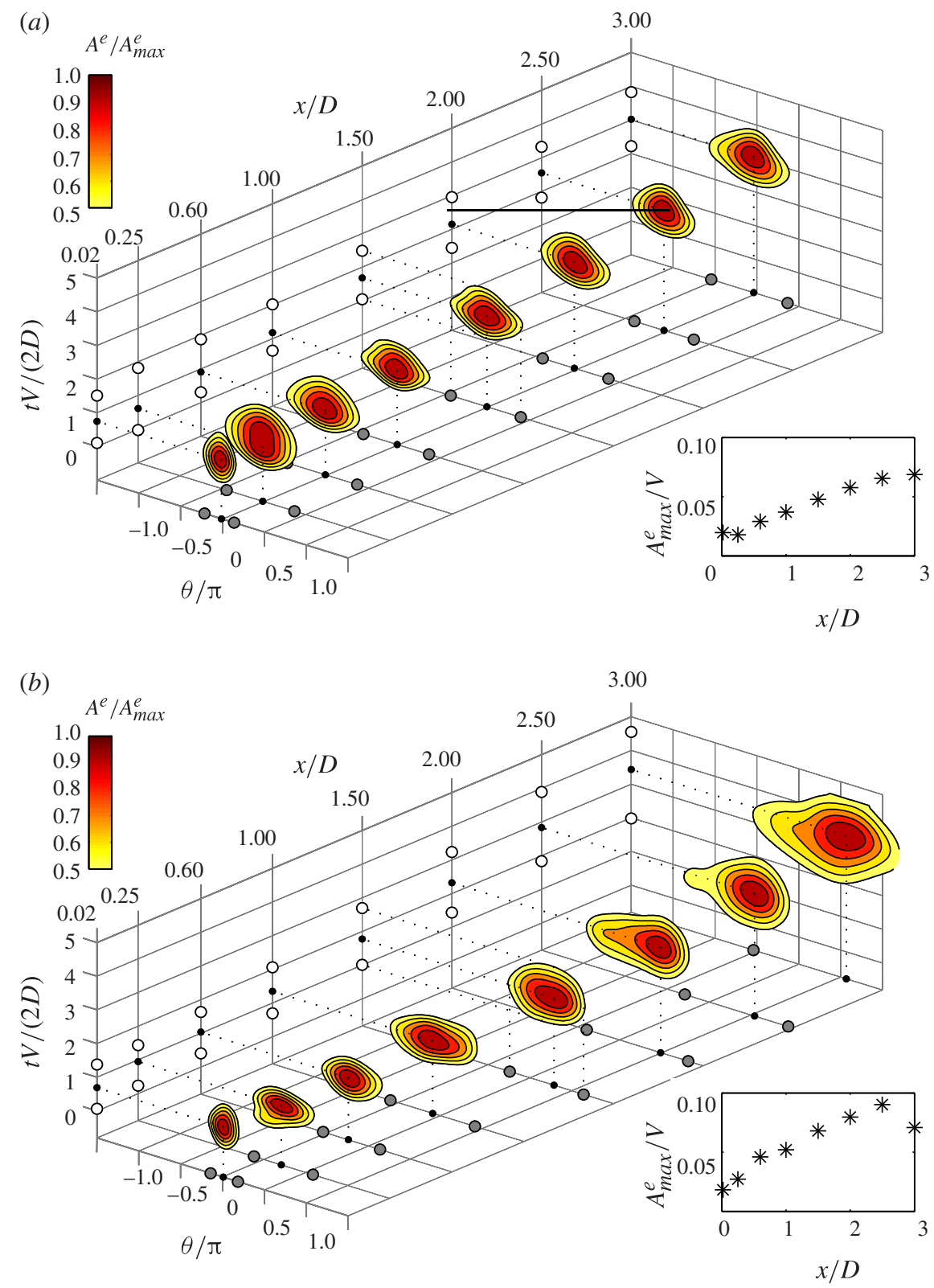

FIgURE 25. (Colour online) Trajectory of the wave packet envelope for the non-swirling jet $S_{1}(a)$ and swirling jet $S_{3}(b)$ derived from hot-wire measurements. The pulse is initiated at $\theta=0, t=0$ and $x / D=0$. Contours show the envelope amplitude distribution $A^{e}$ at each $r-\theta$ plane of measurement normalized by the corresponding maximum $A_{\max }^{e}$. Big black dots refer to the maximum of the envelope projected onto the $\theta-x$ and $t-x$ planes. White-filled black circles refer to the leading and trailing edge of the wave packet in the time direction. Grey-filled black circles refer to the leading and trailing edge of the wave packet in $\theta$ direction. 
circles in the $x-t$ plane and by grey-filled black circles in the $x-\theta$ plane. The centre of the wave packet is associated with $A^{e} / A_{\max }^{e}=1$. Its coordinate is marked on the axis planes by big black dots. The streamwise development of $A_{\max }^{e}$ are shown in the small images placed near the bottom-right corner of the figure $25(a, b)$.

To get familiar with this rather complex plotting style the non-swirling jet is discussed first (figure 25a). The pulse is initiated at the nozzle exit at $t=0$ and $-\pi / 8 \leqslant \theta \leqslant \pi / 8$. This creates a wave packet that peaks at $\theta=0$, with an azimuthal extend of $\Delta \theta \pi / 4$ (see the marker in the $\theta-x$ plane at $x / D=0.02$ ). The leading edge arrives at the first measurement position $(x / D=0.02)$ only shortly after $t=0$ followed by the maximum and the trailing edge (see marker in the $x-t$ plane at $x / D=0.02)$. While travelling to the next downstream measurement point $(x / D=0.25)$ the wave packet spreads significantly in azimuthal direction and in time and $A_{\max }^{e}$ decays slightly. The significant deformation of the wave packet near the nozzle is presumable caused by the imperfect forcing. However, with further downstream distance, amplification sets in and the wave packet maximum grows continuously up to a downstream distance of $x / D=2.5$ (see small image in figure $25 a$ ). Within this region of amplification, the envelope maintains its shape remarkably well despite the streamwise variation of the mean flow. This confirms the weak dispersiveness of the shear layer of the non-swirling jet as predicted from the stability analysis. The wave-packet maximum is found to propagate in axial direction at a velocity of approximately $0.7 \mathrm{~V}$ near the nozzle $(0<x / D<0.6)$ and at $0.54 \mathrm{~V}$ for $x / D>0.6$. The lower value, which corresponds to the amplifying region, compares well with the phase velocity derived from the linear theory of $0.5 \mathrm{~V}$ (confer with figure 10).

Figure 25(b) shows the trajectory of the wave packet for the swirling jet $S_{3}$. The envelope at $x / D=0.02$ is very similar to the non-swirling jet, showing equal shape and maximum amplitude $A_{\max }^{e}$. The abrupt spreading of the pulse upstream of $x / D<0.25$ is also observed for the swirling jet, although the widening of the envelope in time direction is less pronounced compared with the non-swirling jet. The streamwise evolution of $A_{\max }^{e}$ does not indicate a region near the nozzle lip where the wave packet decays. In fact, the wave packet is amplified already at the nozzle exit and gains amplitude up to a streamwise distance of $x / D=2.5$. For the swirling jet, the irregularities introduced by the imperfect forcing seem less significant than for the non-swirling jet and amplification sets in at a shorter distance to the nozzle lip.

The wave packet in the swirled shear layer propagates in axial direction and in the direction of the base flow rotation, as indicated by the coordinates of $A_{\max }^{e}$ projected on the $\theta-x$ plane (figure $25 b$ ). The azimuthal propagation velocity, expressed as an azimuthal rotation rate $\Omega^{e}$, is $2 \pi \Omega^{e} D / V \approx 1.1$ within the region $0<x / D=1.5$, which coincides roughly with the potential core region and the region of constant Rossby number (see figure $6 a-b$ ). With further downstream distance, $\Omega^{e}$ decays rapidly, yielding $2 \pi \Omega^{e} D / V \approx 0.1$ at $x / D=3$. It is interesting to note that the theoretically derived azimuthal phase velocity $\omega_{r} / m$ of the streamwise modes $\left(\alpha_{r}=0\right)$ with $m=2$ and $m=3$ agrees quite well with the rotation rate of the wave packet envelope. This is consistent with the kinematic relation $\omega_{r} \propto V / 2 \alpha_{r}^{\max }+\Omega_{g r} m$ derived in $\S 5$, if one interprets $\Omega^{e}$ as the group rotation rate $\Omega_{g r}$. The streamwise propagation velocity of the envelope is very similar to the non-swirling jet, ranging from $0.7 \mathrm{~V}$ near the nozzle to $0.5 \mathrm{~V}$ further downstream $(x / D>0.6)$. This seems plausible, as the stability analysis predicts modes with low azimuthal wavenumbers, which are dominant further downstream, to have a similar axial phase velocity as for the non-swirling jet. 
However, a prediction of the wave packet propagation speed based on the phase velocities of the individual modes remains cumbersome for the swirling jet due to the dispersiveness of the shear layers. The latter is indicated by a strong deformation of the wave-packet envelope during its downstream propagation. In the region of strong amplitude gain $(0.6 \leqslant x / D \leqslant 2.5)$, the envelope looses its symmetry and its maximum is shifted closer to the leading azimuthal bound, revealing a steep front and a smooth tail of the disturbance envelope in the $\theta$ direction. This pattern results from the superposition of modes with different azimuthal wavenumbers travelling at different streamwise phase velocities, as predicted by the linear theory. In contrast, the wave packet is not deformed significantly in the $t$ direction while travelling downstream. The most unstable modes have small azimuthal wavenumbers $(m<3)$ and small inclination angles $\alpha_{r} / m$ and, thus, different phase velocities result in strong amplitude variations in the $\theta$ direction but only weak variations in the $t$ direction.

\subsection{Modal decomposition of the wave packet}

The measured coherent axial velocity $v_{x}^{c}(x, r, \theta, t)$ is transformed into Fourier space, yielding the coefficients $\hat{v}_{x}^{c}\left(x, r, m, \omega_{r}\right)$. The modal amplitude distribution $A_{m}\left(x, \omega_{r}\right)$ is then derived by integrating $\hat{v}_{x}$ across the shear layer in accordance with (2.3). The same quantity is derived form the linear stability for various $\omega_{r}$ and $m$ utilizing (4.5).

The hot-wire measurements are presented in figure 26 showing contours of $A_{m}$ in the $m-\omega_{r}$ plane at several streamwise measurement locations. It can be directly compared with figure 27 showing the corresponding numerical predictions. For the non-swirling jet, the agreement between measurements and theoretical prediction is good downstream of $x / D=1$. The measurements confirm that the wave packet in the non-swirling shear layer is first dominated by axisymmetric waves at frequencies around $\omega_{r} \delta_{x} / V=0.1$ (see the second frame in the left column). Further downstream, the bending $m= \pm 1$ modes become most dominant. The normalized frequency of highest amplitude remains constant with downstream distance, confirming that the most amplified wavelengths in the packet scales with the local length scale $\delta_{x}$. The mismatch between theoretical prediction and experiment at $x / D \leqslant 1$ is probably attributed to the imperfect forcing and to spatially decaying modes at low frequencies that are excited at the nozzle.

For the swirling jet, the mode selection is in line with the theoretical prediction. Note that the sense of winding of the helical waves cannot be derived from the measurements and the modes can only be classified as co-rotating or counter-rotating modes, which corresponds in figures 26-27 to $m>0$ and $m<0$, respectively. This implies a special caution in interpreting these figures, since for the swirling jet, modes with the same sense of winding may have negative or positive $m$. This is the case for the co-winding double-helical mode that is most amplified within the nearfield. Its maximum amplitude is located at a frequency of $\omega_{r} \delta_{x} / V \approx 0.06$ at $m=-2$, yielding that this mode is counter-rotating. In agreement with the theoretical prediction, the dimensionless frequency of the most unstable mode is reduced with the addition of swirl. The experiments further show that the co-winding double-helical mode can be counter-rotating $\left(\omega_{r}>0, m=-2\right)$, steady $\left(\omega_{r}=0, m= \pm 2\right)$ or co-rotating $\left(\omega_{r}>0, m=2\right)$. Hence, the existence of steady modes at considerably high amplitude are confirmed by the present experiments. Moreover, the measurements conducted downstream of $x / D=2$ indicate the amplification of the single-helical counter-rotating mode in good agreement with the theoretical prediction. 
(a)

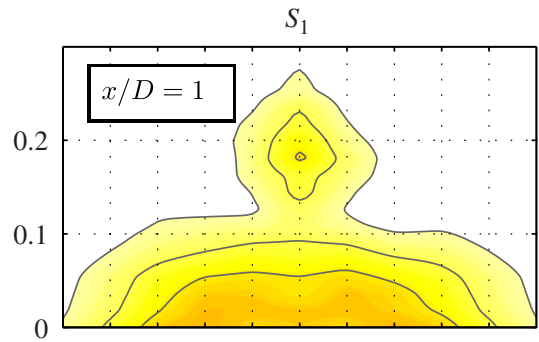

(b)

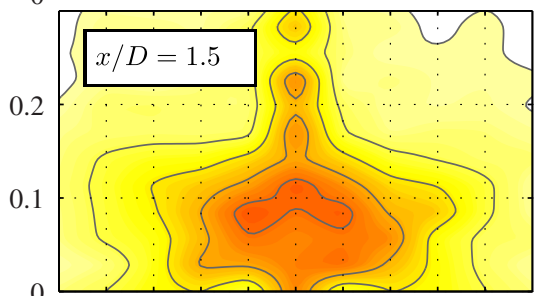

(c)

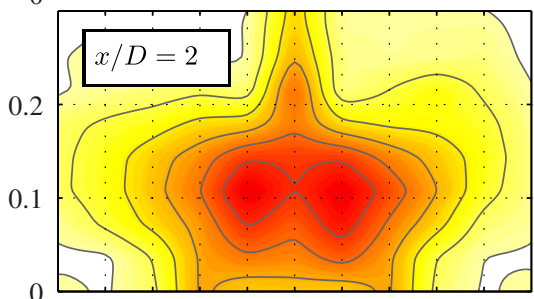

(d)

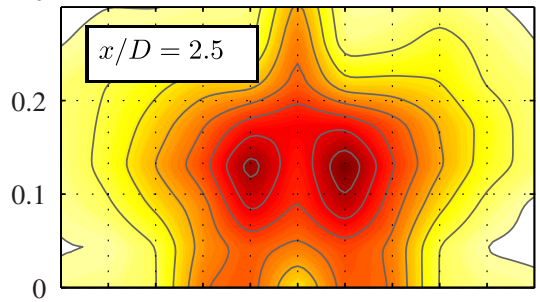

(e)

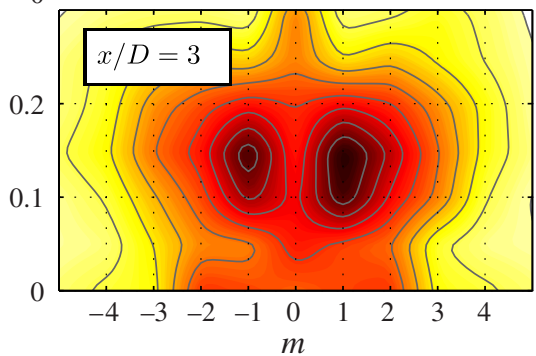

$(f)$

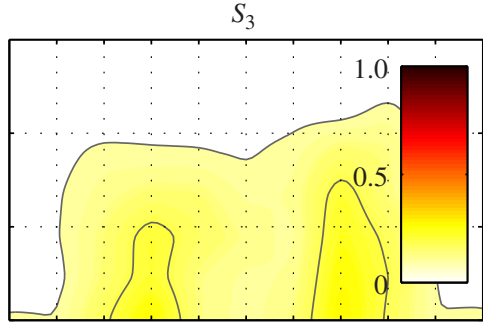

$(g)$

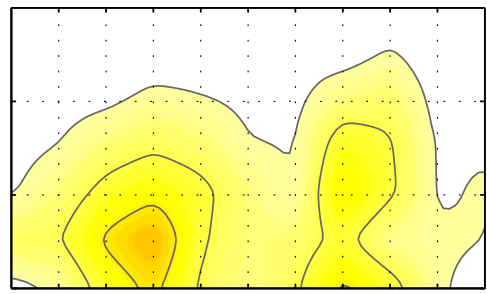

(h)

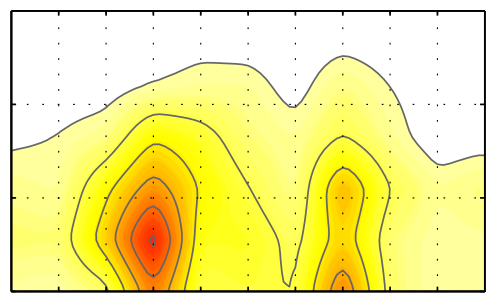

(i)

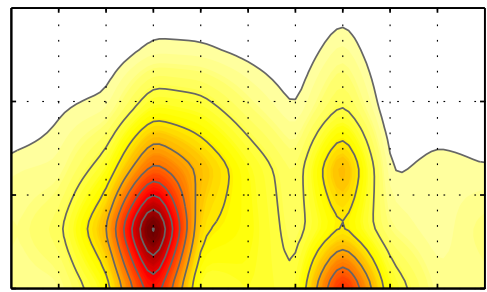

(j)

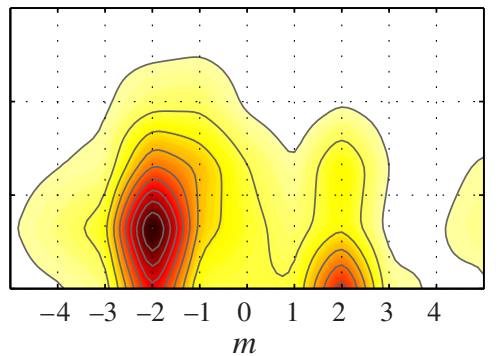

FIGURE 26. (Colour online) Modal amplitude distribution derived from hot-wire measurements: $(a-e)$ non-swirling jet $S_{1}$ and $(f-j)$ swirling jet $S_{3}$; contours refer to the modal amplitude $A_{m}$ normalized by its overall maximum.

\section{Summary and discussion}

\subsection{The purpose of the present investigation}

The nearfield of a turbulent swirling jet is investigated at a Reynolds number of $R e_{D}=20000$. Four different swirl intensities are considered, ranging from zero swirl to intensities that are just below the onset of vortex breakdown. The work 
(a)

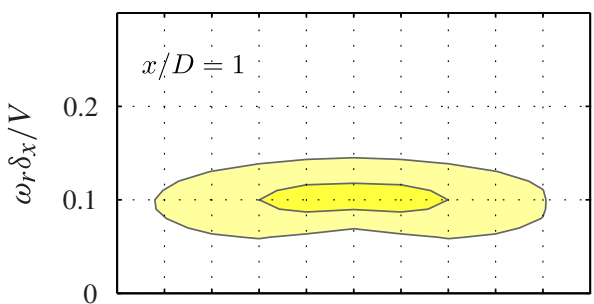

(b)

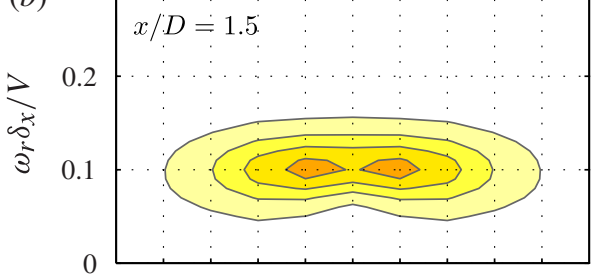

(c)
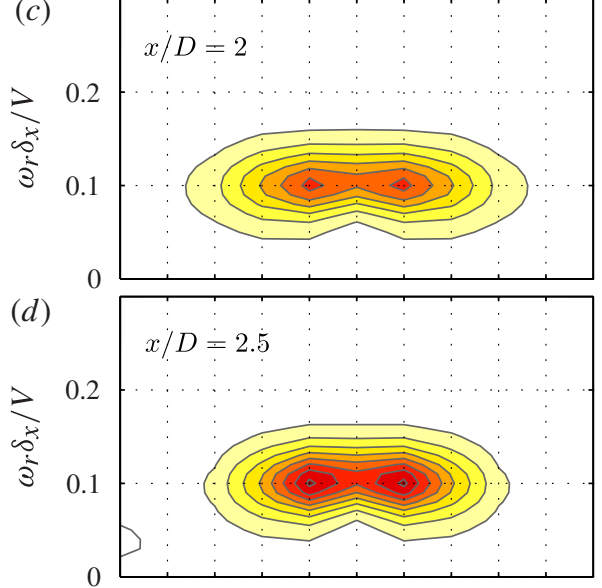

(e)

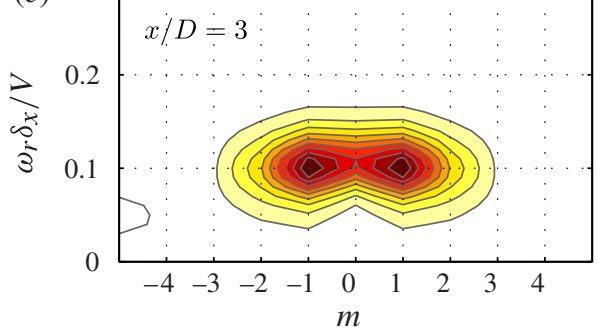

(f) $S_{3}$

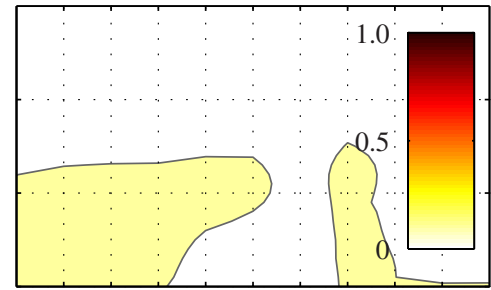

(g)

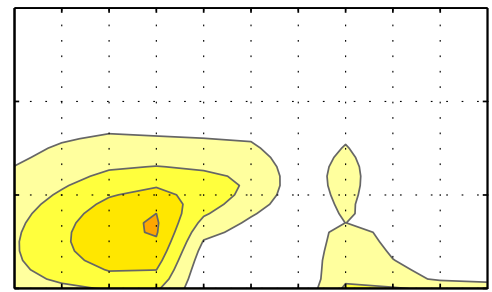

(h)

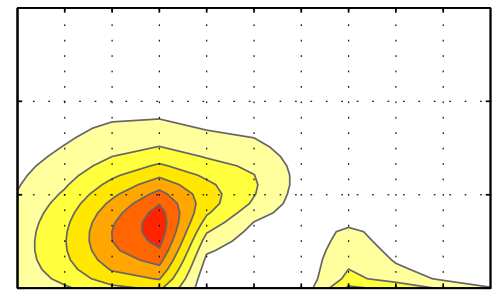

(i)

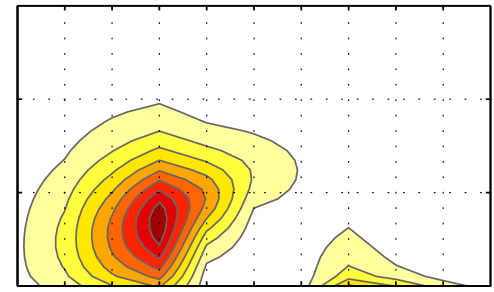

(j)

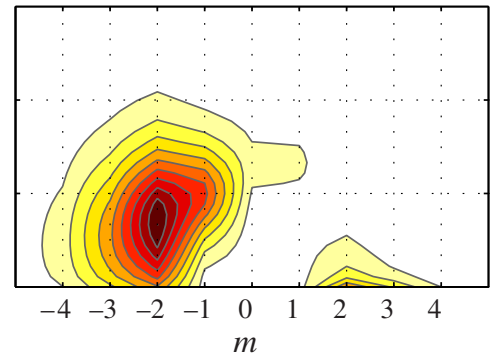

FIgURE 27. (Colour online) Modal amplitude distribution derived from linear stability analysis: $(a-e)$ non-swirling jet $S_{1}$ and $(f-j)$ swirling jet $S_{3}$; contours refer to the modal amplitude $A_{m}$ normalized by its overall maximum.

focuses on the impact of swirl on the dominant flow instabilities that lead to the streamwise formation of coherent structures. The problem is approached theoretically by means of spatial linear stability analysis. Based on the natural mean flow, this method provides the streamwise growth and saturation of the inherent coherent structures. The theoretical results are verified through a quantitative comparison with measurements of the excited flow. 


\subsection{The main observations}

The swirling jet promotes a centrifugal instability and a shear instability. The centrifugal instability scales with the swirl component, destabilizing chiefly co-winding counter-rotating modes at high frequency and wavenumbers. The corresponding overall amplification is weak and all modes decay shortly downstream of the nozzle exit. In contrast, the shear instability prevails over the entire jet nearfield amplifying coand counter-winding modes. Their growth rates scale inversely with the local axial shear-layer thickness, which implies that the enhanced jet spreading encountered at higher swirl decreases their overall amplification. The streamwise wavelengths of the most amplified shear modes are determined by the axial shear-layer thickness regardless of the swirl velocity, however, their frequencies are modified by the base flow rotation. This leads to a destabilization of slowly rotating and even steady modes that undergo significant amplification. The interplay of these selection principles renders the swirling jet's shear layer as highly dispersive for non-axisymmetric modes, unlike the non-swirling jet. The mode with maximum overall amplification, the preferred mode, is strongly affected by swirl. At the end of the potential core, the co-winding $m=2$ mode is dominant for the swirling jet in replacement for the bending $m= \pm 1$ mode of the non-swirling jet. Downstream of the potential core, the $m=2$ mode stabilizes and the co-winding $m=1$ mode takes over the lead, presumably dominating the swirling jet's farfield.

\subsection{Discussion}

The characteristics of the centrifugal and the shear instabilities encountered presently are similar to those found in swirled shear layers (Lu \& Lele 1999; Cooper \& Peake 2002; Müller \& Kleiser 2008). For the present study, the two types of instabilities occur always as two individual wavenumber branches and are considered as two modes driven by two different mechanisms. This allows the instabilities to be phenomenologically separated into centrifugal modes that exist only for the swirling jet and shear modes that are primarily driven by the strong shear of the axial velocity profile. However, it seems to not always be possible to strictly distinguish between centrifugal and shear instabilities. Gallaire \& Chomaz (2003b) applied linear stability analysis to a jet with a thicker axial shear layer than the present one, revealing only one perturbation mode that is a clear Kelvin-Helmholtz instability for no swirl, while it comprises characteristics of a centrifugal instability for strong swirl. It is possibly attributed to the strong differences between the axial and azimuthal shear layer that no instabilities of mixed type are encountered in the present study.

The rapid streamwise decay of the centrifugal instability confirms the analysis of Cooper \& Peake (2002), who investigated the stability of a swirled shear layer that spreads radially due to viscosity. However, they found the shear mode to be significantly amplified by the addition of swirl, which contradicts the present findings. In the present flow, only the growth rates of the centrifugal modes increase with increasing swirl, while the growth rates of the shear modes remain constant. The contraction used in the present experiment generates an inflow profile with a very thin axial shear layer and a relatively thick azimuthal shear layer. This explains the low impact of the azimuthal shear on the growth rate of the shear instability. The inflow profiles used by Lu \& Lele (1999), Cooper \& Peake (2002) and Müller \& Kleiser (2008) reveal much stronger azimuthal shear.

Panda \& McLaughlin (1994) were the first to excite instabilities in the turbulent shear layer, encountering low amplification when compared with the non-swirling jets. 
They concluded that the low receptivity of the swirling jet is attributed to the rapid growth of the axial shear layer, without providing the required experimental evidence. In this study, we confirm their conclusions showing that the axial shear-layer thickness scales the growth rates encountered in turbulent swirling jets. Furthermore, the swirlenhanced dispersiveness of the swirled shear layer, found in the present investigation, explains the absence of vortex pairing as it was observed by Panda \& McLaughlin (1994).

The cause for the dispersiveness of the swirling jet has already been identified by Martin \& Meiburg (1994) as a purely kinematic effect of the mean flow rotation. As outlined in more detail in the present work, the rotational motion implies a non-zero azimuthal group velocity that results in the dependence of the streamwise wavelength and the phase velocity on the azimuthal wavenumber. In other words, the inclination of a helical wave at a given frequency is altered with increasing swirl due to an azimuthal propagation velocity of disturbances in the swirled shear layer. The wave crests of modes at different frequencies or azimuthal wavenumbers travel at different streamwise velocities, which is not the case for the non-swirling jet. The strong dispersiveness in swirling jets hampers the use of effective flow control methods that utilize intermodal resonance principles (Long \& Petersen 1992; Paschereit et al. 1995).

As reexamined in this work, the non-swirling jet is unstable to a large wavenumber and frequency band at the nozzle exit that reduces to the bending $m= \pm 1$ modes downstream of the potential core. The mode selection of the swirling jet is more complex as symmetry breaks down and co-rotating modes undergo different amplification than counter-rotating modes. The presently found results are in line with model-based studies that indicate that swirl tends to destabilize co-winding modes and to stabilize counter-winding modes (see e.g. Loiseleux, Chomaz \& Huerre 1998; Gallaire \& Chomaz 2003a). It is worth noting here that the dominance of the axisymmetric mode observed in swirling and non-swirling jet experiments (see e.g. Ho \& Gutmark 1987; Loiseleux \& Chomaz 2003; Liang \& Maxworthy 2005) is usually attributed to an accidental axisymmetric forcing caused by the facility upstream of the contraction and must not be mistaken with the preferred mode (Cohen \& Wygnanski $1987 a$ ).

Moreover, swirl is found to destabilize two rather exotic types of shear modes, namely the streamwise modes with $\alpha_{r}=0$ and the steady modes with $\omega=0$. The first is purely driven by azimuthal shear revealing relatively small growth rates due to the thick azimuthal shear layer. The steady modes, however, are driven by the axial shear and azimuthal shear, which allows for significant spatial growth. In fact, co-winding double- and triple-helical modes reveal significant amplification rates at frequencies around zero. Hot-wire measurements of a wave packet excited at the nozzle consistently confirm the existence of steady and weakly rotating double-helical modes. Consistent with our observations, steady and nearly steady co-winding spiral modes with $m=2$ or $m=3$ have been observed in experimental arrangements similar to the present one (Billant et al. 1998; Loiseleux \& Chomaz 2003; Oberleithner, Paschereit \& Wygnanski 2007). Gallaire \& Chomaz (2003b) assign the weakly rotating $m=2$ mode observed by Loiseleux \& Chomaz (2003) to a self-excited mode that arises from a convective/absolute transition point near the nozzle exit. In other words, they associate the preferred mode with a globally unstable mode with its wavemaker located near the nozzle exit. The absence of absolute instability in the present flow suggests that the appearance of double- or triple-helical structures, as reported by Billant et al. (1998) or Loiseleux \& Chomaz (2003), correspond to convectively 
unstable steady or nearly steady modes. Their spatial phase may be tagged by a small irregularity of the experimental arrangement, which leads to a breaking of rotational symmetry of the mean flow. Moreover, for very high swirl below breakdown, Loiseleux \& Chomaz (2003) observe a counter-rotating single-helical mode at high rotation rates (frequencies). This mode presumably corresponds to the presently found preferred mode of the farfield.

The present stability analysis does not indicate any mechanism explaining the encountered swirl-enhanced jet spreading. However, the present results may support or cancel one or another recent arguments. Vortex merging, the nonlinear mechanism that leads to a successive shear layer spreading in the potential core region of a non-swirling jet (Ho \& Gutmark 1987; Gutmark et al. 1995), does not occur (or hardly occurs) in swirling jets due to the strong dispersiveness of the axial shear layer. Moreover, the idea of a significant swirl-induced destabilization of centrifugal and/or shear modes that would intensify the growth of coherent structures and enhance the entrainment rates (see e.g. Mehta et al. 1991; Wu, Farokhi \& Taghavi 1992; Panda \& McLaughlin 1994; Cutler et al. 1995; Lu \& Lele 1999; Cooper \& Peake 2002) is not supported by the present work. Even at the nozzle exit, where swirl is strongest, the centrifugal instability is found to be much weaker than the shear mode driven by the axial velocity profile. Hence, the encountered swirl-enhanced jet spreading is unlikely to be attributed to the small change in the base flow stability found at the nozzle exit.

\section{Acknowledgements}

We would like to thank P. Kuhn for conducting the hot-wire measurements reported in this work. This work was financially supported by the German Research Foundation (DFG) and by a fellowship within the Postdoc-Program of the German Academic Exchange Service (DAAD).

\section{REFERENCES}

BARKLEY, D. 2006 Linear analysis of the cylinder wake mean flow. EPL (Europhys. Lett.) 75, $750-756$.

Billant, P., Chomaz, J.-M. \& Huerre, P. 1998 Experimental study of vortex breakdown in swirling jets. J. Fluid Mech. 376, 183-219.

CARTon, X. \& MCWilliams, J. 1989 Barotropic and baroclinic instabilties of axisymmetric vortices in a quasi-geostrophic model. In Mesoscale/Synoptic Coherent Structures in Geophysical Turbulence (ed. J. Nihoul \& B. Jamart), pp. 225-244. Elsevier.

Cohen, J. \& Wygnanski, I. 1987a The evolution of instabilities in the axisymmetric jet. Part 1. The linear growth of disturbances near the nozzle. J. Fluid Mech. 176, 191-219.

Cohen, J. \& Wygnanski, I. $1987 b$ The evolution of instabilities in the axisymmetric jet. Part 2. The flow resulting from the interaction between two waves. J. Fluid Mech. 176, 221-235.

Cooper, A. J. \& PeAKe, N. 2002 The stability of a slowly diverging swirling jet. J. Fluid Mech. 473, 389-411.

Crighton, D. G. \& Gaster, M. 1976 Stability of slowly diverging jet flow. J. Fluid Mech. 77, 397-413.

Cutler, A. D., Kraus, D. K. \& Levey, B. S. 1995 Near-field flow of supersonic swirling jets. AIAA J. 33, 876-881.

Delbende, I., Chomaz, J.-M. \& Huerre, P. 1998 Absolute/convective instabilities in the Batchelor vortex: a numerical study of the linear impulse response. J. Fluid Mech. 355, 229-254.

Drazin, P. G. \& REID, W. H. 2004 Hydrodynamic Stability. Cambridge Mathematical Library. 
Escudier, M. \& Keller, J. 1983 Vortex breakdown: a two-stage transition. In AGARD CP no.324: aerodynamics of vortical type flows in $3 D$. Paper 25.

Gallaire, F. \& Chomaz, J.-M. $2003 a$ Instability mechanisms in swirling flows. Phys. Fluids 15, 2622-2639.

Gallaire, F. \& Chomaz, J.-M. $2003 b$ Mode selection in swirling jet experiments: a linear stability analysis. J. Fluid Mech. 494, 223-253.

Gaster, M. \& Grant, I. 1975 An experimental investigation of the formation and development of a wave packet in a laminar boundary layer. Royal Soc. Lond. Proc. Ser. A 347, 253-269.

Gaster, M., Kit, E. \& WygnAnski, I. 1985 Large-scale structures in a forced turbulent mixing layer. J. Fluid Mech. 150, 23-39.

Greenblatt, D. \& Wygnanski, I. J. 2000 The control of flow separation by periodic excitation. Prog. Aeronaut. Sci. 36 (7), 487-545.

Gudmundsson, K. \& Colonius, T. 2011 Instability wave models for the near-field fluctuations of turbulent jets. J. Fluid Mech. 689, 97-128.

Gutmark, E. J., Schadow, K. C. \& YU, K. H. 1995 Mixing enhancement in supersonic free shear flows. Annu. Rev. Fluid Mech. 27, 375-417.

Herbert, T. 1997 Parabolized stability equations. Annu. Rev. Fluid Mech. 29, 245-283.

Ho, C.-M. \& GutMark, E. 1987 Vortex induction and mass entrainment in a small-aspect-ratio elliptic jet. J. Fluid Mech. 179, 383-405.

HowARD, L. N. \& GUPTA, A. S. 1962 On the hydrodynamic and hydromagnetic stability of swirling flows. J. Fluid Mech. 14, 463-476.

HU, G.-H., Sun, D.-J. \& YIN, X.-Y. 2001a A numerical study of dynamics of a temporally evolving swirling jet. Phys. Fluids 13, 951-965.

Hu, G.-H., Sun, D.-J., YIN, X.-Y. \& BIngGang, T. $2001 b$ Studies on stability and dynamics of a swirling jet. Acta Mechanica Sin. 17, 237-244.

HuAng, Y. \& YANG, V. 2009 Dynamics and stability of lean-premixed swirl-stabilized combustion. Prog. Energy Combust. Sci. 35 (4), 293-364.

Huerre, P. \& Monkewitz, P. A. 1990 Local and global instabilities in spatially developing flows. Annu. Rev. Fluid Mech. 22, 473-537.

Hussain, A. K. M. F. \& ReYnolds, W. C. 1970 The mechanics of an organized wave in turbulent shear flow. J. Fluid Mech. 41, 241-258.

Juniper, M. P., TAmmisola, O. \& Lundell, F. 2011 The local and global stability of confined planar wakes at intermediate Reynolds number. J. Fluid Mech. 686, 218-238.

Khorrami, M. R., Malik, M. R. \& Ash, R. L. 1989 Application of spectral collocation techniques to the stability of swirling flows. J. Comput. Phys. 81 (1), 206-229.

Knowles, K. \& Saddington, A. J. 2006 A review of jet mixing enhancement for aircraft propulsion applications. Proc. IMechE G: J. Aerospace Engng 220 (2), 103-127.

Leibovich, S. \& Stewartson, K. 1983 A sufficient condition for the instability of columnar vortices. J. Fluid Mech. 126, 335-356.

Liang, H. \& Maxworthy, T. 2005 An experimental investigation of swirling jets. J. Fluid Mech. 525, 115-159.

LiU, J. T. C. 1971 Nonlinear development of an instability wave in a turbulent wake. Phys. Fluids 14, 2251-2257.

Loiseleux, T. \& Chomaz, J.-M. 2003 Breaking of rotational symmetry in a swirling jet experiment. Phys. Fluids 15, 511-523.

Loiseleux, T., Chomaz, J. M. \& Huerre, P. 1998 The effect of swirl on jets and wakes: linear instability of the Rankine vortex with axial flow. Phys. Fluids 10, 1120-1134.

Loiseleux, T., Delbende, I. \& Huerre, P. 2000 Absolute and convective instabilities of a swirling jet/wake shear layer. Phys. Fluids 12, 375-380.

Long, T. A. \& Petersen, R. A. 1992 Controlled interactions in a forced axisymmetric jet. Part 1. The distortion of the mean flow. J. Fluid Mech. 235, 37-55.

LU, G. \& LELE, S. K. 1999 Inviscid instability of compressible swirling mixing layers. Phys. Fluids 11, 450-461.

Malik, M. R., Zang, T. A. \& Hussaini, M. Y. 1985 A spectral collocation method for the Navier-Stokes equations. J. Comput. Phys. 61, 64-88. 
Marasli, B., Champagne, F. H. \& Wygnanski, I. J. 1991 On linear evolution of unstable disturbances in a plane turbulent wake. Phys. Fluids 3, 665-674.

Martin, J. E. \& Meiburg, E. 1994 On the stability of the swirling jet shear layer. Phys. Fluids 6, 424-426.

Martin, J. E. \& Meiburg, E. 1996 Nonlinear axisymmetric and three-dimensional vorticity dynamics in a swirling jet model. Phys. Fluids 8 (7), 1917-1928.

Martin, J. E. \& Meiburg, E. 1998 The growth and nonlinear evolution of helical perturbations in a swirling jet model. Eur. J. Mech. (B/Fluids) 17, 639-651.

McManus, K. R., Poinsot, T. \& Candel, S. M. 1993 A review of active control of combustion instabilities. Prog. Energy Combust. Sci. 19, 1-29.

Mehta, R. D., Wood, D. H. \& Clausen, P. D. 1991 Some effects of swirl on turbulent mixing layer development. Phys. Fluids 3, 2716-2724.

Meliga, P., Pujals, G. \& ÉRIC, S. 2012 Sensitivity of 2-D turbulent flow past a D-shaped cylinder using global stability. Phys. Fluids 24 (6), 061701.

Michalke, A. 1965 On spatially growing disturbances in an inviscid shear layer. J. Fluid Mech. 23, 521-544.

Monkewitz, P. A. \& Sohn, K. D. 1988 Absolute instability in hot jets. AIAA J. 26, 911-916.

MüLLER, S. B. \& KLEISER, L. 2008 Viscous and inviscid spatial stability analysis of compressible swirling mixing layers. Phys. Fluids 20 (11), 114103.

Naughton, J. W., Cattafesta, L. N. III \& Settles, G. S. 1997 An experimental study of compressible turbulent mixing enhancement in swirling jets. J. Fluid Mech. 330, 271-305.

Noack, B. R., Afanasiev, K., Morzyński, M., Tadmor, G. \& Thiele, F. 2003 A hierarchy of low-dimensional models for the transient and post-transient cylinder wake. J. Fluid Mech. 497, 335-363.

Oberleithner, K., Paschereit, C. O., Seele, R. \& Wygnanski, I. 2012 Formation of turbulent vortex breakdown: intermittency, criticality, and global instability. AIAA J. 50, 1437-1452.

Oberleithner, K., Paschereit, C. O.\& Wygnanski, I. 2007 Vortex breakdown in a swirling jet with axial forcing. In 18eme Congres Francais de Mechanique, colloquium: Stratified and Rotating Flows. AFM.

Oberleithner, K., Sieber, M., Nayeri, C. N., Paschereit, C. O., Petz, C., Hege, H.-C., NOACK, B. R. \& WygNANSKI, I. 2011 Three-dimensional coherent structures in a swirling jet undergoing vortex breakdown: stability analysis and empirical mode construction. J. Fluid Mech. 679, 383-414.

Olendraru, C. \& Sellier, A. 2002 Viscous effects in the absolute convective instability of the Batchelor vortex. J. Fluid Mech. 459, 371-396.

Panda, J. \& Mclaughlin, D. K. 1994 Experiments on the instabilities of a swirling jet. Phys. Fluids 6, 263-276.

Paschereit, C. O., Wygnanski, I. \& Fiedler, H. E. 1995 Experimental investigation of subharmonic resonance in an axisymmetric jet. J. Fluid Mech. 283, 365-407.

PIER, B. 2002 On the frequency selection of finite-amplitude vortex shedding in the cylinder wake. J. Fluid Mech. 458, 407-417.

Raffel, M., Willert, C., Wereley, S.\& Kompenhans, J. 2007 Particle Image Velocimetry, A Practical Guide. 2nd edn. Springer.

Reau, N. \& Tumin, A. 2002 Harmonic Perturbations in Turbulent Wakes. AIAA J. 40, 526-530.

SARPKAYA, T. 1971 On stationary and travelling vortex breakdowns. J. Fluid Mech. 45, 545-559.

SemaAn, R., NAUghton, J. W. \& EwING, D. 2009 Approach toward similar behaviour of a swirling jet flow. In 47th AIAA Aerospace Sciences Meeting. AIAA.

Townsend, A. 1956 The Structure of Turbulent Shear Flow. Cambridge University Press.

Trefethen, 2000 Spectral Methods in Matlab. SIAM.

Wu, C., FARokhi, S. \& TAghaVI, R. 1992 Spatial instability of a swirling jet - theory and experiment. AIAA J. 30, 1545-1552.

Wu, J.-Z., MA, H.-Y. \& Zhou, M.-D. 2006 Vorticity and Vortex Dynamics. Springer.

Zhou, M. D., Heine, C. \& Wygnanski, I. 1996 The effects of excitation on the coherent and random motion in a plane wall jet. J. Fluid Mech. 310, 1-37. 